\title{
A BANACH SPACE INDUCED BY AN ALMOST DISJOINT FAMILY, ADMITTING ONLY FEW OPERATORS AND DECOMPOSITIONS
}

\author{
PIOTR KOSZMIDER AND NIELS JAKOB LAUSTSEN
}

\begin{abstract}
We consider the closed subspace of $\ell_{\infty}$ generated by $c_{0}$ and the characteristic functions of elements of an uncountable, almost disjoint family $\mathcal{A}$ of infinite subsets of $\mathbb{N}$. This Banach space has the form $C_{0}\left(K_{\mathcal{A}}\right)$ for a locally compact Hausdorff space $K_{\mathcal{A}}$ that is known under many names, including $\Psi$-space and Isbell-Mrówka space.

We construct an uncountable, almost disjoint family $\mathcal{A}$ such that the algebra of all bounded linear operators on $C_{0}\left(K_{\mathcal{A}}\right)$ is as small as possible in the precise sense that every bounded linear operator on $C_{0}\left(K_{\mathcal{A}}\right)$ is the sum of a scalar multiple of the identity and an operator that factors through $c_{0}$ (which in this case is equivalent to having separable range). This implies that $C_{0}\left(K_{\mathcal{A}}\right)$ has the fewest possible decompositions: whenever $C_{0}\left(K_{\mathcal{A}}\right)$ is written as the direct sum of two infinite-dimensional Banach spaces $\mathcal{X}$ and $\mathcal{Y}$, either $\mathcal{X}$ is isomorphic to $C_{0}\left(K_{\mathcal{A}}\right)$ and $\mathcal{Y}$ to $c_{0}$, or vice versa. These results improve previous work of the first named author in which an extra set-theoretic hypothesis was required. We also discuss the consequences of these results for the algebra of all bounded linear operators on our Banach space $C_{0}\left(K_{\mathcal{A}}\right)$ concerning the lattice of closed ideals, characters and automatic continuity of homomorphisms.

To exploit the perfect set property for Borel sets as in the classical construction of an almost disjoint family by Mrówka, we need to deal with $\mathbb{N} \times \mathbb{N}$ matrices rather than with the usual partitioners of an almost disjoint family. This noncommutative setting requires new ideas inspired by the theory of compact and weakly compact operators and the use of an extraction principle due to van Engelen, Kunen and Miller concerning Borel subsets of the square.
\end{abstract}

\section{INTRODUCTION}

The symbols $[\mathbb{N}]^{\omega}$ and $[\mathbb{N}]^{<\omega}$ denote the families of all infinite and finite subsets, respectively, of the set $\mathbb{N}=\{0,1,2 \ldots\}$ of nonnegative integers. A family $\mathcal{A} \subseteq[\mathbb{N}]^{\omega}$ is called almost disjoint if $A \cap A^{\prime} \in[\mathbb{N}]^{<\omega}$ whenever $A, A^{\prime} \in \mathcal{A}$ are distinct.

We shall investigate the impact that the combinatorial structure of an almost disjoint family $\mathcal{A}$ has on the Banach space $\mathcal{X}_{\mathcal{A}}$ associated with it. The formal definition of this space is as follows.

2010 Mathematics Subject Classification. 03E05, 46E15, 47L10, 54D80 (primary); 46B26, 46H40, 47B38, 47L20, 54D45.

Key words and phrases. Banach space of continuous functions, compact Hausdorff space, $\Psi$-space, almost disjoint family, partitioner, perfect set property, bounded linear operator, closed operator ideal.

The main part of the research presented in this paper was carried out while the first named author visited Lancaster University in the early months of 2019. He wishes to thank the second named author for hosting him and the Faculty of Science and Technology of Lancaster University for supporting the visit through the Distinguished Visitor Programme Fund. 
Definition 1. Let $\mathcal{A} \subseteq[\mathbb{N}]^{\omega}$ be an almost disjoint family. Then $\mathcal{X}_{\mathcal{A}}$ is the closed subspace of $\ell_{\infty}$ spanned by $\left\{1_{A}: A \in \mathcal{A} \cup[\mathbb{N}]^{<\omega}\right\}$.

Here, and elsewhere, $1_{A}$ stands for the characteristic function of a set $A$. Banach spaces of the form $\mathcal{X}_{\mathcal{A}}$ were first considered by Johnson and Lindenstrauss in Example 2 of [27]. Being a self-adjoint subalgebra of $\ell_{\infty}, \mathcal{X}_{\mathcal{A}}$ is isometrically isomorphic to $C_{0}\left(K_{\mathcal{A}}\right)$, where $K_{\mathcal{A}}$ is the Gelfand space of $\mathcal{X}_{\mathcal{A}}$. Moreover, $C_{0}\left(K_{\mathcal{A}}\right)$ contains a complemented copy of $c_{0}$, so $C_{0}\left(K_{\mathcal{A}}\right)$ is isomorphic to the Banach space $C\left(\alpha K_{\mathcal{A}}\right)$ of all continuous scalar-valued functions on the one-point compactification $\alpha K_{\mathcal{A}}$ of $K_{\mathcal{A}}$.

The study of $K_{\mathcal{A}}$ as an interesting example of a scattered, locally compact Hausdorff space, which is nonmetrizable whenever $\mathcal{A}$ is uncountable, can be traced back to Alexandroff and Urysohn [3]. Spaces of the form $K_{\mathcal{A}}$ are known under many names, including $\Psi$-spaces, Isbell-Mrówka spaces, AU-compacta and Mrówka spaces. We refer to [24] and [23] for recent surveys on these spaces and their numerous applications found during many decades of investigations.

It is often assumed in the literature that the almost disjoint family $\mathcal{A}$ inducing the space $K_{\mathcal{A}}$ is maximal with respect to inclusion, which corresponds to $K_{\mathcal{A}}$ being pseudocompact. We do not make this assumption here, and indeed the families that we obtain are not maximal.

In [34], the first named author used the continuum hypothesis, or its weakening $\mathfrak{p}=2^{\omega}$, to construct an uncountable, almost disjoint family $\mathcal{A} \subseteq[\mathbb{N}]^{\omega}$ such that every bounded linear operator on $C_{0}\left(K_{\mathcal{A}}\right)$ has the form $\lambda \operatorname{Id}+S$, where $\lambda$ is a scalar, Id denotes the identity operator on $C_{0}\left(K_{\mathcal{A}}\right)$, and $S$ is an operator that factors through $c_{0}$ in the sense that $S=V U$ for some bounded linear operators $U: C_{0}\left(K_{\mathcal{A}}\right) \rightarrow c_{0}$ and $V: c_{0} \rightarrow C_{0}\left(K_{\mathcal{A}}\right)$. Here we provide another construction of such a family $\mathcal{A}$ that does not require any additional set-theoretic axioms.

Theorem 2. There is an uncountable, almost disjoint family $\mathcal{A} \subseteq[\mathbb{N}]^{\omega}$ such that every bounded linear operator $T: C_{0}\left(K_{\mathcal{A}}\right) \rightarrow C_{0}\left(K_{\mathcal{A}}\right)$ has the form

$$
T=\lambda \operatorname{Id}+S
$$

for some scalar $\lambda$ and some operator $S: C_{0}\left(K_{\mathcal{A}}\right) \rightarrow C_{0}\left(K_{\mathcal{A}}\right)$ that factors through $c_{0}$.

Note in particular that the operator $S$ has separable range. In fact, for operators on a space of the form $C_{0}\left(K_{\mathcal{A}}\right)$, it is well known that having separable range and factoring through $c_{0}$ are equivalent properties; see Lemma 22 for details.

Theorem 2 answers a question raised in [34], and together with results of Argyros and Raikoftsalis [5], Marciszewski and Pol [42], and Cabello Sánchez, Castillo, Marciszewski, Plebanek and Salguero-Alarcón [9], it completes the list of solutions to the problems left open in [34].

Theorem 2 has some remarkable consequences, notably:

(i) Whenever the Banach space $C_{0}\left(K_{\mathcal{A}}\right)$ is decomposed into a direct sum $C_{0}\left(K_{\mathcal{A}}\right)=\mathcal{X} \oplus \mathcal{Y}$ of two closed, infinite-dimensional subspaces $\mathcal{X}$ and $\mathcal{Y}$, either $\mathcal{X}$ is isomorphic to $C_{0}\left(K_{\mathcal{A}}\right)$ and $\mathcal{Y}$ is isomorphic to $c_{0}$, or vice versa (see Lemma 4 of [34]).

(ii) The Banach algebra $\mathscr{B}\left(C_{0}\left(K_{\mathcal{A}}\right)\right)$ of all bounded linear operators on the Banach space $C_{0}\left(K_{\mathcal{A}}\right)$ contains precisely four closed two-sided ideals, namely

$$
\{0\} \subsetneq \mathscr{K}\left(C_{0}\left(K_{\mathcal{A}}\right)\right) \subsetneq \mathscr{X}\left(C_{0}\left(K_{\mathcal{A}}\right)\right) \subsetneq \mathscr{B}\left(C_{0}\left(K_{\mathcal{A}}\right)\right),
$$


where $\mathscr{K}\left(C_{0}\left(K_{\mathcal{A}}\right)\right)$ and $\mathscr{X}\left(C_{0}\left(K_{\mathcal{A}}\right)\right)$ are the ideals of compact operators and operators with separable range, respectively. This result is due to Kania and Kochanek (see Theorem 5.5 of [28]) and was also observed by Brooker (unpublished).

(iii) The Banach algebra $\mathscr{B}\left(C_{0}\left(K_{\mathcal{A}}\right)\right)$ admits a character, that is, a nonzero multiplicative linear functional, whose kernel is $\mathscr{X}\left(C_{0}\left(K_{\mathcal{A}}\right)\right)$; we shall discuss this point in much more detail in Subsection 6.3.

(iv) If $\phi: \alpha K_{\mathcal{A}} \rightarrow \alpha K_{\mathcal{A}}$ is a continuous map, then either $\phi$ has countable range or all but countably many points of $\alpha K_{\mathcal{A}}$ are fixed by $\phi$ (see Proposition 36).

Roughly speaking, these results say that $C_{0}\left(K_{\mathcal{A}}\right)$ is "minimal" in terms of its decompositions, closed operator ideals, kernels of characters on $\mathscr{B}\left(C_{0}\left(K_{\mathcal{A}}\right)\right)$ and continuous maps on $\alpha K_{\mathcal{A}}$. Indeed, $C_{0}(K)$ contains a complemented copy of $c_{0}$ whenever $K$ is a scattered, locally compact Hausdorff space, so decompositions of $C_{0}\left(K_{\mathcal{A}}\right)$ of the form described in (i) are unavoidable, as is the ideal $\mathscr{X}\left(C_{0}\left(K_{\mathcal{A}}\right)\right)$ of operators factoring through $c_{0}$ lying strictly between $\mathscr{K}\left(C_{0}\left(K_{\mathcal{A}}\right)\right)$ and $\mathscr{B}\left(C_{0}\left(K_{\mathcal{A}}\right)\right)$ in (ii); and Proposition 44 will show that the kernel of the character on $\mathscr{B}\left(C_{0}\left(K_{\mathcal{A}}\right)\right)$ described in (iii) is as small as it can be.

We note that the conclusion of Theorem 2 and its consequences (i), (ii) and (iii) also hold true if $C_{0}\left(K_{\mathcal{A}}\right)$ is replaced with $C\left(\alpha K_{\mathcal{A}}\right)$ because the two spaces are isomorphic.

Our construction of the almost disjoint family $\mathcal{A}$ will exploit a well-known approach due to Mrówka [45] that starts from a Borel almost disjoint family $\mathcal{A} \subseteq[\mathbb{N}]^{\omega}$ and then takes advantage of the perfect set property for Borel sets. However, in the noncommutative setting of operators rather than continuous functions, instead of the usual partitioners of an almost disjoint family, we need to consider $\mathbb{N} \times \mathbb{N}$ matrices representing operators. The behaviour of such a matrix on the set $A \cup B$ for $A, B \in \mathcal{A}$ is not completely determined by its behaviour on $A$ and $B$ alone, as it also depends on the entries of the matrix belonging to $(A \backslash B) \times(B \backslash A)$ and $(B \backslash A) \times(A \backslash B)$. Overcoming these obstacles requires us to find a new approach, which is inspired by the theory of compact and weakly compact operators on $c_{0}$ and $\ell_{\infty}$, and also to work with Borel subsets of Cartesian products.

Organization. The paper is organized as follows: we begin by recalling the main topological properties of spaces of the form $K_{\mathcal{A}}$ in Section 2, before we sketch the key parts of Mrówka's original argument that our construction is modelled after. In the remainder of Section 2 we introduce some basic notions and establish Theorem 2 subject to a sequence of lemmas, whose proofs are then given in Sections 3,4 and 5 .

Theorem 2 and its consequences interact with a considerable body of known results concerning set theory, logic, topology, Banach spaces and Banach algebras. We discuss this in detail in Section 6, including the structure of continuous self-maps of $\alpha K_{\mathcal{A}}$ and the minimality of the kernel of the character on $\mathscr{B}\left(C_{0}\left(K_{\mathcal{A}}\right)\right)$ mentioned in items (iv) and (iii) above, as well as the automatic continuity of homomorphisms from $\mathscr{B}\left(C_{0}\left(K_{\mathcal{A}}\right)\right)$ into a Banach algebra and the place of our Banach space $C_{0}\left(K_{\mathcal{A}}\right)$ with few operators among other well-known Banach spaces with few operators. Section 6 also contains some open questions which arise naturally from our results, including a discussion of their context and motivation.

Notation and terminology. Our notation and terminology is mostly standard. We list the key parts here. None of our arguments concerning Banach spaces depend 
on whether the space is over the real or complex numbers, so the scalar field will be denoted generically by $\mathbb{K}$. The Banach algebra of all bounded linear operators on a Banach space $\mathcal{X}$ is denoted $\mathscr{B}(\mathcal{X})$, and the identity operator on $\mathcal{X}$ is $\operatorname{Id} \mathcal{X}$, or simply Id if $\mathcal{X}$ is clear from the context. We write $\mathcal{X} \sim \mathcal{Y}$ to signify that the Banach spaces $\mathcal{X}$ and $\mathcal{Y}$ are isomorphic.

All topological spaces that we consider are Hausdorff. For a locally compact Hausdorff space $K, C_{0}(K)$ denotes the Banach space of all $\mathbb{K}$-valued continuous functions $f$ on $K$ such that the set $\{x \in K:|f(x)| \geq \varepsilon\}$ is compact for each $\varepsilon>0$, endowed with the supremum norm. By $1_{A}$ we mean the characteristic function of a set $A$. This notation is slightly ambiguous as the domain of the function $1_{A}$ depends on the ambient set of $A$; it should always be clear from the context. We write $f \mid X$ for the restriction of a function $f$ to a subset $X$ of its domain.

The Cantor cube is denoted by $2^{\mathbb{N}}$. It is the set of all sequences of zeros and ones, and is a compact Hausdorff space with respect to the product topology. For a subset $A$ of $\mathbb{N}$, we may naturally regard $1_{A}$ as an element of $2^{\mathbb{N}}$.

Given a scalar sequence $\left(a_{n}\right)_{n \in \mathbb{N}}$ and a set $A \in[\mathbb{N}]^{\omega}$, we write $\lim _{n \in A} a_{n}=b$ to mean that the sequence $\left(a_{n_{k}}\right)_{k \in \mathbb{N}}$ converges and has limit $b$, where $\left(n_{k}\right)_{k \in \mathbb{N}}$ is the increasing enumeration of $A$.

The symbol $\mathfrak{c}$ will stand for the cardinality of the continuum.

\section{Outline of the Proof of Theorem 2}

In this section we shall prove Theorem 2 subject to a sequence of lemmas whose proofs will then be given in the subsequent sections of the paper. The purpose of this organization is to give a clear overview of the proof before we go into the details. We work with the Banach space $\mathcal{X}_{\mathcal{A}}$ using its representation as a space of the form $C_{0}\left(K_{\mathcal{A}}\right)$ for a locally compact Hausdorff space $K_{\mathcal{A}}$, so we begin by recalling the details of this representation.

2.1. The locally compact space $K_{\mathcal{A}}$ induced by an almost disjoint family $\mathcal{A}$. Recall that the Banach space $\mathcal{X}_{\mathcal{A}}$ was defined in Definition 1.

Definition 3. Let $\mathcal{A} \subseteq[\mathbb{N}]^{\omega}$ be an almost disjoint family. Then $K_{\mathcal{A}}$ denotes the topological space consisting of distinct points $\left\{x_{n}: n \in \mathbb{N}\right\} \cup\left\{y_{A}: A \in \mathcal{A}\right\}$, where $x_{n}$ is isolated for every $n \in \mathbb{N}$ and the sets

$$
U(A, F)=\left\{x_{n}: n \in A \backslash F\right\} \cup\left\{y_{A}\right\}
$$

for $F \in[\mathbb{N}]^{<\omega}$ form a neighbourhood basis at each point $y_{A}$ for $A \in \mathcal{A}$. We write $U(A)$ for $U(A, \emptyset)$.

The following lemma summarizes well known consequences of standard general topological results (see [24, 23]).

Lemma 4. Let $\mathcal{A} \subseteq[\mathbb{N}]^{\omega}$ be an almost disjoint family. Then:

- $K_{\mathcal{A}}$ is a locally compact, scattered Hausdorff space.

- $K_{\mathcal{A}}$ is compact if and only if $\mathcal{A}$ and $\mathbb{N} \backslash \bigcup \mathcal{A}$ are both finite.

- $\left\{x_{n}: n \in \mathbb{N}\right\}$ is the set of isolated points of $K_{\mathcal{A}}$; it is dense in $K_{\mathcal{A}}$, and so $K_{\mathcal{A}}$ is separable. Hence $K_{\mathcal{A}}$ is metrizable if and only it is second countable, if and only if $\mathcal{A}$ is countable.

- The subspace $K_{\mathcal{A}} \backslash\left\{x_{n}: n \in \mathbb{N}\right\}=\left\{y_{A}: A \in \mathcal{A}\right\}$ is closed and discrete.

- The sequence $\left(x_{n}\right)_{n \in A}$ converges to $y_{A}$ in $K_{\mathcal{A}}$ for every $A \in \mathcal{A}$. 
Lemma 5. The Banach space $\mathcal{X}_{\mathcal{A}}$ is isometric to the space of all continuous functions on $K_{\mathcal{A}}$ vanishing at infinity.

Proof. Consider the operator $T: C_{0}\left(K_{\mathcal{A}}\right) \rightarrow \ell_{\infty}$ defined by $T(f)(n)=f\left(x_{n}\right)$ for each $n \in \mathbb{N}$. We have $T\left(1_{U(A, F)}\right)=1_{A \backslash F} \in \mathcal{X}_{\mathcal{A}}$ and $T\left(1_{\left\{x_{n}\right\}}\right)=1_{\{n\}} \in \mathcal{X}_{\mathcal{A}}$. All compact open sets of $K_{\mathcal{A}}$ are finite unions of sets of the form $U(A, F)$ and finite subsets of $\left\{x_{n}: n \in \mathbb{N}\right\}$, so the range of $T$ is $\mathcal{X}_{\mathcal{A}}$. By the density of $\left\{x_{n}: n \in \mathbb{N}\right\}$ in $K_{\mathcal{A}}$, the operator $T$ is an isometry.

2.2. Constructing $C_{0}\left(K_{\mathcal{A}}\right)$ with few multiplication operators. This subsection is not needed for obtaining the proofs of any results in the remainder of this paper. That is why the proofs are omitted or sketched. We provide this subsection as a motivation for the next one. It is an extraction of some ideas from [34] (cf. [24]), which are later modified. The main result of this subsection is Theorem 12 where the existence of an almost disjoint family $\mathcal{B} \subseteq[\mathbb{N}]^{\omega}$ is proved such that $K_{\mathcal{B}}$ has few bounded continuous scalar-valued functions, so in particular $C_{0}\left(K_{\mathcal{B}}\right)$ has few multiplication operators. In the next subsection we show how to modify these ideas to obtain $C_{0}\left(K_{\mathcal{B}}\right)$ with few operators.

The main idea is to consider all potential bounded continuous functions $F$ on $K_{\mathcal{B}}$ by representing them as elements $f$ of $\ell_{\infty}$ given by $f(n)=F\left(x_{n}\right)$. We may enumerate these elements as $\left\{f_{\xi}: \xi<\mathfrak{c}\right\}$. The family $\mathcal{B}=\left\{B_{\xi}: \xi<\mathfrak{c}\right\}$ is constructed by a transfinite induction of length $\mathfrak{c}$, making sure that $B_{\xi}$ rejects $f_{\xi}$, i.e., it witnesses that $f_{\xi}$ does not represent a bounded continuous function on $K_{\mathcal{B}}$ unless $f_{\xi}$ is of the form mentioned in Theorem 12. This can be achieved, for example, by choosing $B_{\xi}$ such that $\lim _{k \in B_{\xi}} f_{\xi}(k)$ does not exist, because $\lim _{k \in B_{\xi}} F\left(x_{k}\right)$ must exist and be equal to $F\left(y_{B_{\xi}}\right)$ for every continuous function $F: K_{\mathcal{B}} \rightarrow \mathbb{K}$ since $\left(x_{k}\right)_{k \in B_{\xi}}$ converges to $y_{B_{\xi}}$ in $K_{\mathcal{B}}$ by Lemma 4 .

It is useful to introduce the following terminology:

Definition 6. Suppose that $\mathcal{A} \subseteq[\mathbb{N}]^{\omega}$ and $A \in[\mathbb{N}]^{\omega}$. We say that:

- $A$ admits $f \in \ell_{\infty}$ if $\lim _{n \in A} f(n)=0$.

- $\mathcal{A}$ admits $f$ if $A$ admits $f$ for every $A \in \mathcal{A}$.

- $A$ rejects $f \in \ell_{\infty}$ if $\lim _{n \in A} f(n)$ does not exist.

- $\mathcal{A}$ rejects $f$ if $A$ rejects $f$ for some $A \in \mathcal{A}$.

Lemma 7. Suppose that $f \in \ell_{\infty}$ and that $A, A^{\prime} \in[\mathbb{N}]^{\omega}$.

(1) (Monotonicity) If $\left\{A, A^{\prime}\right\}$ admits $f$, then $A \cup A^{\prime}$ admits $f$.

(2) (Decidability) If $A$ does not admit $f-\lambda 1_{\mathbb{N}}$ for any $\lambda \in \mathbb{K}$, then $A^{\prime}$ rejects $f$ for every $A^{\prime} \supseteq A$.

The following lemma explains what properties of an almost disjoint family in terms of rejection and admission are needed to obtain Theorem 12.

Lemma 8 (Reduction). Suppose that $\mathcal{B} \subseteq\left[\mathbb{N}^{\omega}\right]^{\omega}$ is an almost disjoint family such that, for every $f \in \ell_{\infty}$, either $\mathcal{B}$ rejects $f$ or there is $\lambda_{f} \in \mathbb{K}$ and a countable subset $\mathcal{B}_{f} \subseteq \mathcal{B}$ such that $\mathcal{B} \backslash \mathcal{B}_{f}$ admits $f-\lambda_{f} 1_{\mathbb{N}}$. Then all bounded continuous functions $F: K_{\mathcal{B}} \rightarrow \mathbb{K}$ are of the form

$$
F=\lambda 1_{K_{\mathcal{B}}}+G,
$$

where $\lambda \in \mathbb{K}$ and $G$ is nonzero only on countably many points of $K_{\mathcal{B}}$.

Proof. Let $F: K_{\mathcal{B}} \rightarrow \mathbb{K}$ be a bounded continuous function, and let $f \in \ell_{\infty}$ be given by $f(n)=F\left(x_{n}\right)$. If $f$ was rejected by $\mathcal{B}$ as witnessed by $B \in \mathcal{B}$, this would 
contradict the continuity of $F$ at $y_{B}$ as $\lim _{k \in B} x_{k}=y_{B}$ in $K_{\mathcal{B}}$ by Lemma 4 , so by the property of $\mathcal{B}$, there is a countable $\mathcal{B}_{f} \subseteq \mathcal{B}$ and $\lambda_{f} \in \mathbb{K}$ such that $\mathcal{B} \backslash \mathcal{B}_{f}$ admits $f-\lambda_{f} 1_{\mathbb{N}}$. This means that $\lim _{k \in B} f(k)=\lambda_{f}$ for all $B \in \mathcal{B} \backslash \mathcal{B}_{f}$, and so for $G=F-\lambda_{f} 1_{K_{\mathcal{B}}}$, we have

$$
G\left(y_{B}\right)=\left(F-\lambda_{f} 1_{K_{\mathcal{B}}}\right)\left(y_{B}\right)=\lim _{k \in B}\left(F-\lambda_{f} 1_{K_{\mathcal{B}}}\right)\left(x_{k}\right)=\lim _{k \in B}\left(f(k)-\lambda_{f}\right)=0
$$

for all but countably many $B \in \mathcal{B}$, which completes the proof the lemma.

To construct $\mathcal{B}=\left\{B_{\xi}: \xi<\mathfrak{c}\right\}$ satisfying the hypothesis of Lemma 8 and consequently Theorem 12, we need to make sure that the transfinite induction can be continued. There are many dangers along the way. One of them is the wellknown fact that it is consistent that there are maximal almost disjoint families of cardinality less than $\mathfrak{c}$. The main idea of how to avoid these dangers is to obtain $\mathcal{B}$ as a simple modification of an uncountable, almost disjoint family which is Borel in the sense of Lemma 30. Such a family is either countable or has cardinality $\mathfrak{c}$ by a powerful classical dichotomy due to Alexandrov and Hausdorff; see Lemma 29 for details.

Before we can execute this strategy, we require a few preparations.

Lemma 9 (Borel set and function induced by $f \in \ell_{\infty}$ ). Let $f \in \ell_{\infty}$. Then

$$
D(f)=\left\{1_{A} \in 2^{\mathbb{N}}: A \text { admits } f-\lambda 1_{\mathbb{N}} \text { for some } \lambda \in \mathbb{K}\right\}
$$

is a Borel subset of $2^{\mathbb{N}}$, and the function $L_{f}: D(f) \rightarrow \mathbb{K}$ given by $L_{f}\left(1_{A}\right)=$ $\lim _{n \in A} f(n)$ is Borel measurable.

Lemma 10 (A dichotomy for Borel functions). Suppose that $X$ is an uncountable Borel subset of $2^{\mathbb{N}}$ and $\phi: X \rightarrow \mathbb{K}$ is Borel measurable. Then either $\phi$ is constant on a cocountable subset of $X$ or there are pairwise disjoint sets $\left\{x_{\xi}, x_{\xi}^{\prime}\right\} \subseteq X$ for $\xi<\mathfrak{c}$ such that $\phi\left(x_{\xi}\right) \neq \phi\left(x_{\xi}^{\prime}\right)$ for every $\xi<\mathfrak{c}$.

Proof. By Lemma 29, $X$ has cardinality c. Consider preimages of singletons under $\phi$, which are Borel subsets of $X$. If all of them are countable, we can easily choose the sets $\left\{x_{\xi}, x_{\xi}^{\prime}\right\}$ by transfinite recursion. Otherwise $Y=\phi^{-1}[\{\lambda\}]$ has cardinality $\mathfrak{c}$ for some $\lambda \in \mathbb{K}$. If $Y$ is a cocountable subset of $X$, we are done. Otherwise $X \backslash Y$ has cardinality $\mathfrak{c}$ by Lemma 29 , and so we can choose $x_{\xi} \in Y$ and $x_{\xi}^{\prime} \in X \backslash Y$ by transfinite recursion.

Combining these two lemmas, we can prove a preparatory dichotomy.

Lemma 11 (Dichotomy for rejection and admission). Suppose that $\mathcal{A} \subseteq[\mathbb{N}]^{\omega}$ is an uncountable, almost disjoint family such that $\left\{1_{A}: A \in \mathcal{A}\right\}$ is a Borel subset of $2^{\mathbb{N}}$, and let $f \in \ell_{\infty}$. Then one of the following holds:

(1) either $\mathcal{A} \backslash \mathcal{A}_{f}$ admits $f-\lambda_{f} 1_{\mathbb{N}}$ for some countable $\mathcal{A}_{f} \subseteq \mathcal{A}$ and $\lambda_{f} \in \mathbb{K}$,

(2) or there are pairwise disjoint $\left\{A_{\xi}, A_{\xi}^{\prime}\right\} \subseteq \mathcal{A}$ for all $\xi<\mathfrak{c}$ such that $A_{\xi} \cup A_{\xi}^{\prime}$ rejects $f$.

Proof. Let $X=\left\{1_{A}: A \in \mathcal{A}\right\} \subseteq 2^{\mathbb{N}}$, and suppose that $X \backslash D(f)$ is uncountable. Then $X \backslash D(f)$ has cardinality $\mathfrak{c}$ by Lemma 29. If $1_{A}, 1_{A^{\prime}} \in X \backslash D(f)$, then by the Decidability of Lemma 7, $A \cup A^{\prime}$ rejects $f$, so any pairwise disjoint family of two-element subsets of $X \backslash D(f)$ satisfies the second alternative of the lemma.

Now suppose that $X \backslash D(f)$ is countable, which means that $X \cap D(f)$ has cardinality $\mathfrak{c}$ by Lemma 29. Lemmas 9 and 10 yield two possibilities for the function 
$\phi=L_{f}: D(f) \rightarrow \mathbb{K}$. If $L_{f}$ is constant on a cocountable subset of $D(f)$, and thus of $X$, we are in the first alternative of the lemma. Otherwise there are pairwise disjoint sets $\left\{1_{A_{\xi}}, 1_{A_{\xi}^{\prime}}\right\} \subseteq X$ such that $L_{f}\left(1_{A_{\xi}}\right) \neq L_{f}\left(1_{A_{\xi}^{\prime}}\right)$ for every $\xi<\mathfrak{c}$. Then the second alternative of the lemma holds as $\lim _{n \in A_{\xi} \cup A_{\xi}^{\prime}} f(n)$ does not exist.

We are now ready to establish the main result:

Theorem 12 (Existence). There is an uncountable, almost disjoint family $\mathcal{B} \subseteq[\mathbb{N}]^{\omega}$ such that all bounded continuous functions $F: K_{\mathcal{B}} \rightarrow \mathbb{K}$ are of the form

$$
F=\lambda 1_{K_{\mathcal{B}}}+G
$$

where $\lambda \in \mathbb{K}$ and $G$ is nonzero on at most countably many points of $K_{\mathcal{B}}$.

Proof. (1) Using Lemma 30, fix an uncountable, almost disjoint family $\mathcal{A} \subseteq$ $[\mathbb{N}]^{\omega}$ such that $\left\{1_{A}: A \in \mathcal{A}\right\}$ is a Borel subset of $2^{\mathbb{N}}$.

(2) Let $\left\{f_{\xi}: \xi<\mathfrak{c}\right\}$ be an enumeration of the set of all elements $f \in \ell_{\infty}$ for which there is no countable $\mathcal{A}_{f} \subseteq \mathcal{A}$ and no $\lambda_{f} \in \mathbb{K}$ such that $\mathcal{A} \backslash \mathcal{A}_{f}$ admits $f-\lambda_{f} 1_{\mathbb{N}}$. (If this set is empty, then Lemma 8 shows that $\mathcal{B}=\mathcal{A}$ already has the required property; if it is nonempty, but has cardinality less than $\mathfrak{c}$, simply repeat each element continuum many times.) Then the second alternative of the dichotomy for admission and rejection (Lemma 11) holds for each $f_{\xi}$ and $\mathcal{A}$.

(3) By transfinite recursion on $\xi<\mathfrak{c}$, construct $A_{\xi}, A_{\xi}^{\prime} \in \mathcal{A}$ and $B_{\xi} \subseteq \mathbb{N}$ such that

(a) $\left\{A_{\eta}, A_{\eta}^{\prime}\right\} \cap\left\{A_{\xi}, A_{\xi}^{\prime}\right\}=\emptyset$ for all $\eta<\xi<\mathfrak{c}$,

(b) $B_{\xi}=A_{\xi} \cup A_{\xi}^{\prime}$ rejects $f_{\xi}$.

(4) Define $\mathcal{B}=\left\{B_{\xi}: \xi<\mathfrak{c}\right\}$.

(5) Check the required properties of $\mathcal{B}$, i.e., that the hypothesis of Lemma 8 holds. Take $f \in \ell_{\infty}$. If $f=f_{\xi}$ for some $\xi<\mathfrak{c}$, then $B_{\xi}$ rejects $f_{\xi}$. Otherwise $\mathcal{A} \backslash \mathcal{A}_{f}$ admits $f-\lambda_{f} 1_{\mathbb{N}}$ for some countable $\mathcal{A}_{f} \subseteq \mathcal{A}$ and some $\lambda_{f} \in \mathbb{K}$, and then the Monotonicity of Lemma 7 and condition (b) above imply that $\mathcal{B} \backslash \mathcal{B}^{\prime}$ admits $f-\lambda_{f} 1_{\mathbb{N}}$ for some countable $\mathcal{B}^{\prime} \subseteq \mathcal{B}$.

2.3. Constructing $C_{0}\left(K_{\mathcal{A}}\right)$ with few operators. Our main construction follows the stages of the construction from Subsection 2.2. Note that the lemmas below will be proved in the following sections and keep their numbering from those sections.

Recall that we used elements of $\ell_{\infty}$ to represent bounded continuous functions on $K_{\mathcal{B}}$. Here we shall use $\mathbb{K}$-valued $\mathbb{N} \times \mathbb{N}$ matrices to represent operators on $C_{0}\left(K_{\mathcal{B}}\right)$.

Definition 13. Let $M=\left(m_{k, n}\right)_{k, n \in \mathbb{N}}$ be an $\mathbb{N} \times \mathbb{N}$ matrix whose entries belong to $\mathbb{K}$. Then:

- $\mathbb{M}=\{M:\|M\|<\infty\}$, where $\|M\|=\sup \left\{\sum_{n \in \mathbb{N}}\left|m_{k, n}\right|: k \in \mathbb{N}\right\}$.

- For $f \in \ell_{\infty}, M f \in \ell_{\infty}$ is given by $(M f)(k)=\sum_{n \in \mathbb{N}} m_{k, n} f(n)$ for $k \in \mathbb{N}$.

- $I$ stands for the $\mathbb{N} \times \mathbb{N}$ matrix which has entries 1 on the diagonal and all remaining entries are 0 .

Definition 14. Suppose that $M \in \mathbb{M}, \mathcal{A} \subseteq[\mathbb{N}]^{\omega}$ and $B \in[\mathbb{N}]^{\omega}$. We say that:

- $\mathcal{A}$ admits $M$ if $\lim _{k \in A^{\prime}}\left(M 1_{A}\right)(k)=0$ for every $A, A^{\prime} \in \mathcal{A}$.

- $B$ rejects $M$ if $\lim _{k \in B}\left(M 1_{B}\right)(k)$ does not exist.

- $B$ undermines $M$ if there is $n \in \mathbb{N}$ such that the sequence $\left(\left(M 1_{\{n\}}\right)(k)\right)_{k \in B}$ does not converge to zero. 
The elements of $\mathbb{M}$ are not as well suited to represent bounded linear operators on $C_{0}\left(K_{\mathcal{B}}\right)$ as the elements of $\ell_{\infty}$ were to represent bounded continuous functions on $K_{\mathcal{B}}$. However, they do represent operators to some extent. We analyze this situation in Section 3 where, in addition to some general results about operators on $C_{0}(K)$ for $K$ scattered, we obtain the following analogue of Lemma 8.

Lemma 23. (Reduction Lemma). Suppose that $\mathcal{B} \subseteq[\mathbb{N}]^{\omega}$ is an almost disjoint family such that, for every $M \in \mathbb{M}$, one of the following three conditions holds:

(1) there are uncountably many $B \in \mathcal{B}$ which reject $M$,

(2) or there are uncountably many $B \in \mathcal{B}$ that undermine $M$,

(3) or there is $\lambda \in \mathbb{K}$ and a countable subset $\mathcal{B}^{\prime} \subseteq \mathcal{B}$ such that $\mathcal{B} \backslash \mathcal{B}^{\prime}$ admits $M-\lambda I$.

Then all bounded linear operators $T: C_{0}\left(K_{\mathcal{B}}\right) \rightarrow C_{0}\left(K_{\mathcal{B}}\right)$ are of the form

$$
T=\lambda \mathrm{Id}+S
$$

where $\lambda \in \mathbb{K}$ and $S$ is an operator which factors through $c_{0}$.

Hence the rest of the efforts of the paper are focused on the construction of an almost disjoint family which satisfies the hypothesis of Lemma 23. If we try to follow the ideas of Subsection 2.2 we quickly realize that the corresponding version of Lemma 7 fails badly for the simple reason that elements of $\mathbb{M}$ need not be monotone. We do not know how to overcome a number of problems stemming from this fact while working with the above notion of admission.

Instead our approach is to consider a version of admission which we call "acceptance" that is sufficiently monotone and can be viewed as a kind of "hereditary admission". This change, on the other hand, complicates other parts of the construction. Our motivation comes from the theory of compact operators on $c_{0}$ and $\ell_{\infty}$ and is explained in more detail at the end of Section 4.

We introduce the following notation and terminology for matrices:

Definition 15. Suppose that $M=\left(m_{k, n}\right)_{k, n \in \mathbb{N}} \in \mathbb{M}$.

- For $j \in \mathbb{N}$, set $M_{j}=\left(m_{k, n}^{\prime}\right)_{k, n \in \mathbb{N}}$, where $m_{k, n}^{\prime}=0$ if $n \leq j$ and $m_{k, n}^{\prime}=m_{k, n}$ otherwise.

- $M$ is called a compact matrix if $\lim _{j \in \mathbb{N}}\left\|M_{j}\right\|=0$.

- For $A \in[\mathbb{N}]^{\omega}$, define $M^{A}=\left(m_{k, n}^{\prime}\right)_{k, n \in \mathbb{N}}$, where $m_{k, n}^{\prime}=m_{k, n}$ if $n, k \in A$ and $m_{k, n}^{\prime}=0$ otherwise.

- We say that $A \in[\mathbb{N}]^{\omega}$ accepts $M$ if $M^{A}$ is a compact matrix.

- We say that $\mathcal{A} \subseteq[\mathbb{N}]^{\omega}$ accepts $M$ if $A \cup A^{\prime}$ accepts $M$ for every $A, A^{\prime} \in \mathcal{A}$.

Section 4 contains the discussion of the relevant operator theoretic aspects of compact matrices and culminates in the proof of the following result:

Lemma 28. Suppose that $M \in \mathbb{M}$ and that $\mathcal{A} \subseteq[\mathbb{N}]^{\omega}$.

(1) (Admission) If $\mathcal{A}$ accepts $M$ and no element of $\mathcal{A}$ undermines $M$, then $\mathcal{A}$ admits $M$.

(2) (Monotonicity) If $\mathcal{A}$ accepts $M$, then $B$ accepts $M$ for every $B \in[\mathbb{N}]^{\omega}$ which is included in a finite union of elements of $\mathcal{A}$.

(3) (Decidability) If $A \in[\mathbb{N}]^{\omega}$ does not accept $M-\lambda I$ for any $\lambda \in \mathbb{K}$, then there is an infinite $B \subseteq A$ such that $B$ rejects $M$. 
(4) (Amalgamation) If, for every $A, A^{\prime} \in \mathcal{A}$, there is $\lambda_{A, A^{\prime}} \in \mathbb{K}$ such that $A \cup A^{\prime}$ accepts $M-\lambda_{A, A^{\prime}} I$, then there is $\lambda \in \mathbb{K}$ such that $\mathcal{A}$ accepts $M-\lambda I$.

In Section 5 we embark on developing some tools which will enable us to take advantage of the Borel structure of $2^{\mathbb{N}}$, beginning with the following lemma.

Lemma 30. There is an uncountable, almost disjoint family $\mathcal{A} \subseteq[\mathbb{N}]^{\omega}$ such that $\left\{1_{A}: A \in \mathcal{A}\right\} \subseteq 2^{\mathbb{N}}$ is closed.

Dealing with operators and matrices brings us into the noncommutative world, where we must consider pairs of indices $A, A^{\prime} \in \mathcal{A}$ (see, e.g., Definitions 14 and 15), rather than single indices as in Subsection 2.2. To handle this situation, we require a Borel dichotomy for pairs.

Lemma 31. (A dichotomy for Borel sets in the square) Suppose that $X \subseteq 2^{\mathbb{N}} \times 2^{\mathbb{N}}$ is a Borel set. Then one of the following conditions holds:

(1) either there is a countable $Y \subseteq 2^{\mathbb{N}}$ such that each point of $X$ has at least one of its coordinates in $Y$,

(2) or there is $Z \subseteq X$ of cardinality continuum such that for any distinct points $(p, q),\left(p^{\prime}, q^{\prime}\right) \in Z$, we have $\{p, q\} \cap\left\{p^{\prime}, q^{\prime}\right\}=\emptyset$.

Lemma 34. Let $M \in \mathbb{M}$. Then

$$
E(M)=\left\{\left(1_{A}, 1_{A^{\prime}}\right) \in 2^{\mathbb{N}} \times 2^{\mathbb{N}}: A \cup A^{\prime} \text { accepts } M-\lambda I \text { for some } \lambda \in \mathbb{K}\right\}
$$

is a Borel subset of $2^{\mathbb{N}} \times 2^{\mathbb{N}}$.

The above two lemmas and Lemma 28 allow us to prove the following analogue of Lemma 11:

Lemma 35. (A dichotomy for acceptance and rejection) Suppose that $M \in \mathbb{M}$ and $\mathcal{A} \subseteq[\mathbb{N}]^{\omega}$ is an uncountable, almost disjoint family such that $\left\{1_{A}: A \in \mathcal{A}\right\}$ is a Borel subset of $2^{\mathbb{N}}$. Then one of the following holds:

(1) either $\mathcal{A} \backslash \mathcal{A}^{\prime}$ accepts $M-\lambda I$ for some countable $\mathcal{A}^{\prime} \subseteq \mathcal{A}$ and $\lambda \in \mathbb{K}$,

(2) or there are pairwise disjoint sets $\left\{A_{\xi}, A_{\xi}^{\prime}\right\} \subseteq \mathcal{A}$ and an infinite subset $B_{\xi} \subseteq A_{\xi} \cup A_{\xi}^{\prime}$ such that $B_{\xi}$ rejects $M$ for every $\xi<\mathfrak{c}$.

This, in particular, means that in the construction of the almost disjoint family satisfying the hypothesis of the Reduction Lemma 23, we need to handle not only unions of pairs as in the proof of Theorem 12, but also their subsets. Fortunately the Monotonicity of Lemma 28 shows that our notion of acceptance allows us to do that, emphasizing how acceptance is a kind of "hereditary admission".

We are now ready to show how our main result can be deduced from the above lemmas.

Theorem 2. There is an uncountable, almost disjoint family $\mathcal{B} \subseteq[\mathbb{N}]^{\omega}$ such that every bounded linear operator $T: C_{0}\left(K_{\mathcal{B}}\right) \rightarrow C_{0}\left(K_{\mathcal{B}}\right)$ has the form $T=\lambda \operatorname{Id}+S$, where $\lambda \in \mathbb{K}$ and $S: C_{0}\left(K_{\mathcal{B}}\right) \rightarrow C_{0}\left(K_{\mathcal{B}}\right)$ factors through $c_{0}$.

Proof. (1) Using Lemma 30, fix an uncountable, almost disjoint family $\mathcal{A} \subseteq$ $[\mathbb{N}]^{\omega}$ such that $\left\{1_{A}: A \in \mathcal{A}\right\}$ is a Borel subset of $2^{\mathbb{N}}$. 
(2) Let $\mathbb{M}^{\prime}$ be the set of all matrices $M \in \mathbb{M}$ such that there is no countable $\mathcal{A}_{M} \subseteq \mathcal{A}$ and no $\lambda_{M} \in \mathbb{K}$ such that $\mathcal{A} \backslash \mathcal{A}_{M}$ accepts $M-\lambda_{M} I$. If $\mathbb{M}^{\prime}=\emptyset$, set $\mathcal{B}=\mathcal{A}$ and go straight to Step (5). Otherwise let $\left\{M_{\xi}: \xi<\mathfrak{c}\right\}$ be an enumeration of $\mathbb{M}^{\prime}$ with each matrix repeated continuum many times, and note that the second alternative of the dichotomy for acceptance and rejection (Lemma 35) holds for each $M_{\xi}$ and $\mathcal{A}$.

(3) By transfinite recursion on $\xi<\mathfrak{c}$, construct $A_{\xi}, A_{\xi}^{\prime} \in \mathcal{A}$ and $B_{\xi} \in[\mathbb{N}]^{\omega}$ such that

(a) $\left\{A_{\eta}, A_{\eta}^{\prime}\right\} \cap\left\{A_{\xi}, A_{\xi}^{\prime}\right\}=\emptyset$ for all $\eta<\xi<\mathfrak{c}$,

(b) $B_{\xi} \subseteq A_{\xi} \cup A_{\xi}^{\prime}$,

(c) $B_{\xi}$ rejects $M_{\xi}$.

(4) Define $\mathcal{B}=\left\{B_{\xi}: \xi<\mathfrak{c}\right\}$.

(5) We check the required properties of $\mathcal{B}$, i.e., that $\mathcal{B}$ satisfies one of the three conditions stated in the Reduction Lemma 23. Fix $M \in \mathbb{M}$.

If $M \in \mathbb{M}^{\prime}$, then there are continuum many $\xi<\mathfrak{c}$ such that $M_{\xi}=M$, and $B_{\xi}$ rejects $M$ for each of these $\xi$, so the first condition in Lemma 23 holds. If the set $\mathcal{B}^{\prime}=\{B \in \mathcal{B}: B$ undermines $M\}$ is uncountable, then the second condition in Lemma 23 holds.

It remains to consider the case where the set $\mathcal{B}^{\prime}$ is countable and $M \notin \mathbb{M}^{\prime}$, so that $\mathcal{A} \backslash \mathcal{A}_{M}$ accepts $M-\lambda_{M} I$ for some countable $\mathcal{A}_{M} \subseteq \mathcal{A}$ and some $\lambda_{M} \in \mathbb{K}$. Note that no element of $\mathcal{B} \backslash \mathcal{B}^{\prime}$ undermines $M-\lambda_{M} I$ because $\left(\lambda_{M} I\right) 1_{\{n\}}=\lambda_{M} 1_{\{n\}}$ for every $n \in \mathbb{N}$. The Monotonicity of Lemma 28 implies that we can find a countable set $\mathcal{B}^{\prime \prime} \subseteq \mathcal{B}$ such that $\mathcal{B} \backslash \mathcal{B}^{\prime \prime}$ accepts $M-\lambda_{M} I$ since all but countably many elements of $\mathcal{B}$ are subsets of unions of pairs of elements of $\mathcal{A} \backslash \mathcal{A}_{M}$. Hence every element of $\mathcal{B} \backslash\left(\mathcal{B}^{\prime} \cup \mathcal{B}^{\prime \prime}\right)$ accepts $M-\lambda_{M} I$ and no element of $\mathcal{B} \backslash\left(\mathcal{B}^{\prime} \cup \mathcal{B}^{\prime \prime}\right)$ undermines $M-\lambda_{M} I$, so the Admission of Lemma 28 implies that $\mathcal{B} \backslash\left(\mathcal{B}^{\prime} \cup \mathcal{B}^{\prime \prime}\right)$ admits $M-\lambda_{M} I$. Therefore the third condition in Lemma 23 holds, and the theorem follows.

Hence we have proved our main Theorem 2 subject to the proofs of Lemmas 23, $28,30,31,34,35$ which will be proved in the following sections.

\section{Bounded linear operators on $C_{0}(K)$ For $K$ Hausdorff, Locally COMPACT AND SCATTERED}

In this section we consider the following standard Banach spaces over the scalar field $\mathbb{K}$, where $\Gamma$ is an arbitrary index set: $\ell_{\infty}(\Gamma)$ consisting of all $f: \Gamma \rightarrow \mathbb{K}$ such that $\|f\|_{\infty}:=\sup _{\gamma \in \Gamma}|f(\gamma)|<\infty$ and $\ell_{1}(\Gamma)$ consisting of all $f: \Gamma \rightarrow \mathbb{K}$ such that $\|f\|_{1}:=\sum_{\gamma \in \Gamma}|f(\gamma)|<\infty$, as well as $c_{0}(\Gamma)$, which is the closure in $\ell_{\infty}(\Gamma)$ of the subspace $c_{00}(\Gamma)$ consisting of finitely supported elements. We shall also consider the collection $\mathbb{M}(\Gamma)$ of $\mathbb{K}$-valued $\Gamma \times \Gamma$ matrices $M=\left(m_{\gamma, \gamma^{\prime}}\right)_{\gamma, \gamma^{\prime} \in \Gamma}$ such that $\sup \left\{\left\|\left(m_{\gamma, \gamma^{\prime}}\right)_{\gamma^{\prime} \in \Gamma}\right\|_{1}: \gamma \in \Gamma\right\}<\infty$. As usual we multiply such a matrix $M$ by an element $f \in \ell_{\infty}(\Gamma)$ to obtain the element $M f \in \ell_{\infty}(\Gamma)$ defined by

$$
(M f)(\gamma)=\left\langle\left(m_{\gamma, \gamma^{\prime}}\right)_{\gamma^{\prime} \in \Gamma}, f\right\rangle=\sum_{\gamma^{\prime} \in \Gamma} m_{\gamma, \gamma^{\prime}} f\left(\gamma^{\prime}\right) .
$$

3.1. Representing operators by infinite matrices. Recall that all topological spaces we consider are assumed to be Hausdorff. A topological space is scattered if 
every (closed) nonempty subset of it has a relatively isolated point. For basic properties of compact scattered spaces, see 8.5 of [54]. The Banach space $C(K)$ for $K$ compact and scattered is characterized by an impressive list of strong conditions, many of which were already surveyed in [48]. For instance, $K$ is scattered if and only if $C(K)$ is an Asplund space (see [15]). Another condition that is equivalent to $K$ being scattered is that the dual space $C(K)^{*}$ is isometric to $\ell_{1}(K)$. This condition is due to Rudin [51] and will play a key role in the following.

If $K$ is locally compact and scattered, then its one-point compactification $\alpha K$ is compact and scattered, and so $C(\alpha K)^{*}$ is isometric to $\ell_{1}(\alpha K)$. By the Riesz representation theorem for locally compact spaces (see, e.g., 18.4.1 of [54]), the space $C_{0}(K)^{*}$ is formed of Radon measures on $\alpha K$ which vanish outside of $K$. This means that $C_{0}(K)^{*}$ is isometric to $\ell_{1}(K)$, and hence a locally compact variant of Rudin's theorem holds as well. We will identify the elements of $C_{0}(K)^{*}$ with Radon measures on $K$ and with elements of $\ell_{1}(K)$ (i.e., absolutely summable functions on $K)$.

For $T \in \mathscr{B}\left(C_{0}(K)\right)$ and $x \in K$, we have $T^{*}\left(\delta_{x}\right)=\sum_{y \in K} T^{*}\left(\delta_{x}\right)(\{y\}) \delta_{y}$, where $T^{*}$ stands for the adjoint of $T$ and $\delta_{x}$ for the probability measure concentrated in $x$, and hence

$$
T(f)(x)=T^{*}\left(\delta_{x}\right)(f)=\sum_{y \in K} T^{*}\left(\delta_{x}\right)(\{y\}) f(y)=\int f d T^{*}\left(\delta_{x}\right)=\left\langle T^{*}\left(\delta_{x}\right), f\right\rangle
$$

for $f \in C_{0}(K)$. This suggests looking at operators on $C_{0}(K)$ as matrices in $\mathbb{M}(K)$.

Definition 16. Suppose that $K$ is a scattered, locally compact Hausdorff space and that $T: C_{0}(K) \rightarrow C_{0}(K)$ is bounded and linear. Then the matrix of $T$ is the $K \times K$ matrix $M_{T}=\left(m_{x, y}\right)_{x, y \in K}$ given by $m_{x, y}=T^{*}\left(\delta_{x}\right)(\{y\})$ for $x, y \in K$.

Lemma 17. Suppose that $K$ is a scattered, locally compact Hausdorff space. Then for every bounded linear operator $T: C_{0}(K) \rightarrow C_{0}(K)$ we have $M_{T} \in \mathbb{M}(K)$, and

$$
T(f)=M_{T} f
$$

for every $f \in C_{0}(K)$.

Proof. We have $M_{T} \in \mathbb{M}(K)$ by Rudin's theorem mentioned above, and $T(f)(x)=$ $\sum_{y \in K} T^{*}\left(\delta_{x}\right)(\{y\}) f(y)=\left(M_{T} f\right)(x)$ for every $f \in C_{0}(K)$ and $x \in K$.

As usual, $K^{\prime}$ denotes the Cantor-Bendixson derivative of a topological space $K$, that is, the subspace of $K$ formed of the nonisolated points of $K$. If $K$ is scattered, then the set of isolated points $K \backslash K^{\prime}$ is dense in $K$ (since isolated points relative to an open set are isolated in $K$ ).

Definition 18. Let $K$ be a scattered, locally compact Hausdorff space. The reduced matrix of a bounded linear operator $T: C_{0}(K) \rightarrow C_{0}(K)$ is $M_{T}^{r}=\left(m_{x, y}\right)_{x, y \in K \backslash K^{\prime}}$, where $m_{x, y}=T^{*}\left(\delta_{x}\right)(\{y\})$ for $x, y \in K \backslash K^{\prime}$, as above.

Any continuous function is, of course, determined by its values on any dense subset of its domain. Analogously, bounded linear operators on $C_{0}(K)$ are, to some extent, determined by their reduced matrix.

Lemma 19. Let $K$ be an infinite, scattered, locally compact Hausdorff space, and let $D=K \backslash K^{\prime}$ be the set of its isolated points. Suppose that $T: C_{0}(K) \rightarrow C_{0}(K)$ 
is bounded and linear. Then there is $E \subseteq K^{\prime}$ of cardinality not bigger than the cardinality of $D$ such that

$$
T(f) \mid D=M_{T}^{r}(f \mid D)
$$

whenever $f \in C_{0}(K)$ and $f(y)=0$ for every $y \in E$.

Proof. Let $E=\left\{y \in K^{\prime}: T^{*}\left(\delta_{x}\right)(\{y\}) \neq 0\right.$ for some $\left.x \in D\right\}$. As $T^{*}\left(\delta_{x}\right) \in \ell_{1}(K)$, for a given $x \in K$, the value $T^{*}\left(\delta_{x}\right)(\{y\})$ is nonzero for at most countably many $y \in K$. Hence the cardinality of $E$ is not bigger than the cardinality of $D$, as $D$ is infinite by the hypothesis that $K$ is infinite. For $x \in D$ and $f \in C_{0}(K)$ with $f(y)=0$ for all $y \in E$, we have

$$
T(f)(x)=\sum_{y \in K \backslash K^{\prime}} T^{*}\left(\delta_{x}\right)(\{y\}) f(y)=\left(M_{T}^{r}(f \mid D)\right)(x),
$$

as required.

A natural question is which $D \times D$ matrices are reduced matrices of bounded linear operators on $C_{0}(K)$, or which $K \times K$ matrices are matrices of bounded linear operators on $C_{0}(K)$. This, of course, depends on the topology of $K$, which determines the space $C_{0}(K) \subseteq \ell_{\infty}(K)$. However, we know that such matrices belong to $\mathbb{M}(D)$ and $\mathbb{M}(K)$, respectively. Using standard arguments, one can show that these matrices correspond exactly to all bounded linear operators from $c_{0}(D)$ into $\ell_{\infty}(D)$ (respectively, from $c_{0}(K)$ into $\ell_{\infty}(K)$ ), and such operators are in isometric correspondence with the weak ${ }^{*}$-continuous operators on $\ell_{\infty}(D)$ (respectively, on $\ell_{\infty}(K)$ ), and with the adjoints of operators on $\ell_{1}(D)$ (respectively, on $\ell_{1}(K)$ ). We refer to [60] for details of these correspondences, which will not be exploited here.

3.2. Bounded linear operators on the Banach space $C_{0}\left(K_{\mathcal{A}}\right)$. The main purpose of this subsection is to prove the Reduction Lemma 23. For this we need a piece of terminology and a couple of lemmas. First recall the terminology and the topological facts concerning the space $K_{\mathcal{A}}$ from Subsection 2.1.

Definition 20. Suppose that $\mathcal{A} \subseteq[\mathbb{N}]^{\omega}$ is an almost disjoint family. For $f \in$ $C_{0}\left(K_{\mathcal{A}}\right)$ and $\mathcal{X} \subseteq C_{0}\left(K_{\mathcal{A}}\right)$, we define

$$
s(f)=\left\{A \in \mathcal{A}: f\left(y_{A}\right) \neq 0\right\}, \quad s(\mathcal{X})=\bigcup\{s(f): f \in \mathcal{X}\} .
$$

Lemma 21. Suppose that $\mathcal{A} \subseteq[\mathbb{N}]^{\omega}$ is an almost disjoint family. A closed subspace $\mathcal{X}$ of $C_{0}\left(K_{\mathcal{A}}\right)$ is separable if and only if $s(\mathcal{X})$ is countable.

Proof. First note that for any $f \in C_{0}\left(K_{\mathcal{A}}\right)$, the set $s(f)$ is countable because only finite subsets of the discrete, closed set $\left\{y_{A}: A \in \mathcal{A}\right\}$ are compact, and $\left\{x \in K_{\mathcal{A}}:|f(x)| \geq \varepsilon\right\}$ is compact for each $\varepsilon>0$.

Now suppose that $\mathcal{X}$ is separable, and let $\mathcal{D}$ be a countable dense subset of $\mathcal{X}$. Then $\mathcal{B}=s(\mathcal{D})$ is countable. Note that $s(\mathcal{X}) \subseteq \mathcal{B}$ because the set

$$
\left\{f \in C\left(K_{\mathcal{A}}\right): s(f) \subseteq \mathcal{B}\right\}=\left\{f \in C\left(K_{\mathcal{A}}\right): f\left(y_{A}\right)=0 \text { for all } A \in \mathcal{A} \backslash \mathcal{B}\right\}
$$

is a closed subspace of $C_{0}\left(K_{\mathcal{A}}\right)$, and it contains $\mathcal{D}$, so it contains $\mathcal{X}$. Hence $s(\mathcal{X})$ is countable.

On the other hand, if $s(\mathcal{X})$ is countable, then $\mathcal{X}$ is isomorphic to a subspace of $C_{0}\left(K_{s(\mathcal{X})}\right)$, which is separable by Lemma 4 . 
Lemma 22 (Example 2c of [27], cf. Lemma 3 of [34]). Suppose that $\mathcal{A} \subseteq[\mathbb{N}]^{\omega}$ is an almost disjoint family and that $\mathcal{X} \subseteq C_{0}\left(K_{\mathcal{A}}\right)$ is separable. Then there is a closed subspace $\mathcal{Y}$ of $C_{0}\left(K_{\mathcal{A}}\right)$ such that $\mathcal{X} \subseteq \mathcal{Y}$ and $\mathcal{Y} \sim c_{0}$.

Recall the terminology introduced in Subsection 2.3, in particular Definitions 13 and 14.

Lemma 23 (Reduction Lemma). Suppose that $\mathcal{B} \subseteq[\mathbb{N}]^{\omega}$ is an almost disjoint family such that, for every $M \in \mathbb{M}$, one of the following three conditions holds:

(1) there are uncountably many $B \in \mathcal{B}$ which reject $M$,

(2) or there are uncountably many $B \in \mathcal{B}$ that undermine $M$,

(3) or there is $\lambda \in \mathbb{K}$ and a countable subset $\mathcal{B}^{\prime} \subseteq \mathcal{B}$ such that $\mathcal{B} \backslash \mathcal{B}^{\prime}$ admits $M-\lambda I$.

Then all bounded linear operators $T: C_{0}\left(K_{\mathcal{B}}\right) \rightarrow C_{0}\left(K_{\mathcal{B}}\right)$ are of the form

$$
T=\lambda \operatorname{Id}+S,
$$

where $\lambda \in \mathbb{K}$ and $S$ is an operator which factors through $c_{0}$.

Proof. Let $K=K_{\mathcal{B}}$, and fix a bounded linear operator $T: C_{0}(K) \rightarrow C_{0}(K)$. Consider its reduced matrix $M_{T}^{r}=\left(m_{x_{k}, x_{n}}\right)_{x_{k}, x_{n} \in K \backslash K^{\prime}}$, and define $M=\left(m_{k, n}\right)_{k, n \in \mathbb{N}}$ by $m_{k, n}=m_{x_{k}, x_{n}}$ for all $k, n \in \mathbb{N}$. Lemma 17 and Definition 18 imply that $M \in \mathbb{M}$.

First we shall see that for this choice of $M$, there are at most countably many elements $B \in \mathcal{B}$ which reject or undermine $M$. This will be based on the part of Lemma 4 which says that $\lim _{k \in B} x_{k}=y_{B}$ for every $B \in \mathcal{B}$. In fact the continuity of $T\left(1_{U(B)}\right)$ at all points $y_{B^{\prime}}$ for $B^{\prime} \in \mathcal{B}$ will contradict rejecting or undermining $M$ for uncountably many elements of $\mathcal{B}$.

As $D=K \backslash K^{\prime}=\left\{x_{n}: n \in \mathbb{N}\right\}$ is countable, by Lemma 19 there is a countable set $E \subseteq K^{\prime}=\left\{y_{B}: B \in \mathcal{B}\right\}$ such that $T(f) \mid D=M_{T}^{r}(f \mid D)$ whenever $f \in C_{0}(K)$ satisfies $f(y)=0$ for every $y \in E$. Let $\mathcal{B}_{1}=\left\{B \in \mathcal{B}: y_{B} \in E\right\}$. The above condition translates into $T\left(1_{U(B)}\right) \mid D=M_{T}^{r}\left(1_{U(B)} \mid D\right)$ for $B \in \mathcal{B} \backslash \mathcal{B}_{1}$, which implies that

$$
T\left(1_{U(B)}\right)\left(y_{B}\right)=\lim _{k \in B} T\left(1_{U(B)}\right)\left(x_{k}\right)=\lim _{k \in B}\left(M_{T}^{r}\left(1_{U(B)} \mid D\right)\right)\left(x_{k}\right)=\lim _{k \in B}\left(M 1_{B}\right)(k) .
$$

Hence no element of $\mathcal{B} \backslash \mathcal{B}_{1}$ rejects $M$, so in particular at most countably many elements of $\mathcal{B}$ reject $M$.

The subspace $\mathcal{X}=\left\{f \in C_{0}(K): f\left(y_{B}\right)=0\right.$ for all $\left.B \in \mathcal{B}\right\}$ is separable because $\left\{1_{\left\{x_{n}\right\}}: n \in \mathbb{N}\right\}$ spans a dense subspace of it. It follows that $T[\mathcal{X}]$ is separable, and therefore $\mathcal{B}_{2}=s(T[\mathcal{X}])$ is countable by Lemma 21 . Let $n, k \in \mathbb{N}$. As $x_{n} \notin K^{\prime}$, Lemma 19 implies that $T\left(1_{\left\{x_{n}\right\}}\right)\left(x_{k}\right)=M_{T}^{r}\left(1_{\left\{x_{n}\right\}} \mid D\right)\left(x_{k}\right)=\left(M 1_{\{n\}}\right)(k)$, and so $\lim _{k \in B}\left(M 1_{\{n\}}\right)(k)=T\left(1_{\left\{x_{n}\right\}}\right)\left(y_{B}\right)=0$ for every $B \in \mathcal{B} \backslash \mathcal{B}_{2}$. This shows that no element of $\mathcal{B} \backslash \mathcal{B}_{2}$ undermines $M$, so in particular at most countably many elements of $\mathcal{B}$ undermine $M$.

Hence the hypothesis about $\mathcal{B}$ implies that there is $\lambda \in \mathbb{K}$ and a countable subset $\mathcal{B}^{\prime} \subseteq \mathcal{B}$ such that $\mathcal{B} \backslash \mathcal{B}^{\prime}$ admits $M-\lambda I$. We shall now complete the proof by showing that the operator $S=T-\lambda \operatorname{Id} \in \mathscr{B}\left(C_{0}(K)\right)$ factors through $c_{0}$. Since $\operatorname{Id}\left(1_{U(B)}\right)\left(x_{k}\right)=1_{U(B)}\left(x_{k}\right)=1_{B}(k)$ for $B \in \mathcal{B}$ and $k \in \mathbb{N}$, the condition that $\mathcal{B} \backslash \mathcal{B}^{\prime}$ admits $M-\lambda I$ implies that

$$
0=\lim _{k \in B^{\prime}}\left((M-\lambda I) 1_{B}\right)(k)=\lim _{k \in B^{\prime}}\left((T-\lambda \operatorname{Id})\left(1_{U(B)}\right)\right)\left(x_{k}\right)=\lim _{k \in B^{\prime}} S\left(1_{U(B)}\right)\left(x_{k}\right)
$$


for $B \in \mathcal{B} \backslash\left(\mathcal{B}_{1} \cup \mathcal{B}^{\prime}\right)$ and $B^{\prime} \in \mathcal{B} \backslash \mathcal{B}^{\prime}$. As $\left(x_{k}\right)_{k \in B^{\prime}}$ converges to $y_{B^{\prime}}$ and $S\left(1_{U(B)}\right)$ is continuous on $K$, this yields that $S\left(1_{U(B)}\right)\left(y_{B^{\prime}}\right)=0$ for $B, B^{\prime}$ as above, and so we have

$$
s\left(\left\{S\left(1_{U(B)}\right): B \in \mathcal{B} \backslash\left(\mathcal{B}_{1} \cup \mathcal{B}^{\prime}\right)\right\}\right) \subseteq \mathcal{B}^{\prime} .
$$

This, together with the fact that the characteristic functions $1_{U(B)}$ and $1_{\left\{x_{n}\right\}}$ for $B \in \mathcal{B}$ and $n \in \mathbb{N}$ span a dense subspace of $C_{0}(K)$, implies that

$$
s\left(S\left[C_{0}(K)\right]\right) \subseteq \mathcal{B}^{\prime} \cup s\left(\left\{S\left(1_{U(B)}\right): B \in \mathcal{B}_{1} \cup \mathcal{B}^{\prime}\right\}\right) \cup s\left(\left\{S\left(1_{\left\{x_{n}\right\}}\right): n \in \mathbb{N}\right\}\right),
$$

which is countable by Lemma 21, and so $S\left[C_{0}(K)\right]$ is separable by another application of Lemma 21. By Lemma 22, the range of $S$ is contained in a copy of $c_{0}$, so $S$ factors through $c_{0}$, as required.

\section{Compact $\mathbb{N} \times \mathbb{N}$ Matrices}

The main purpose of this section is to prove Lemma 28, which is used in both Subsection 2.3 and Section 5. Lemma 26 will also be used in Section 5. The main motivation behind this section is of operator theoretic nature. However, the results that we need are purely combinatorial, so we leave their operator theoretic aspects as a comment at the end of the section. Recall Definitions 13 and 15.

Lemma 24. Suppose that $M=\left(m_{k, n}\right)_{k, n \in \mathbb{N}} \in \mathbb{M}$. Then $M$ is compact if and only if $\lim _{n \rightarrow \infty}\left(\sum_{i \in F_{n}} m_{k_{n}, i}\right)=0$ whenever $\left(F_{n}\right)_{n \in \mathbb{N}} \subseteq[\mathbb{N}]<\omega$ are pairwise disjoint and $\left(k_{n}\right)_{n \in \mathbb{N}} \subseteq \mathbb{N}$ is strictly increasing.

Proof. It is clear that if $M$ is compact, then the condition of the lemma holds because $\left|\sum_{i \in F_{n}} m_{k_{n}, i}\right| \leq\left\|M_{\min \left(F_{n}\right)-1}\right\|$, and $\min \left(F_{n}\right) \rightarrow \infty$ as $n \rightarrow \infty$ since the sets $F_{n}$ are pairwise disjoint.

Conversely, suppose that $M$ is not compact. Then there is $\varepsilon>0$ such that $\left\|M_{j}\right\|>\varepsilon$ for infinitely many $j \in \mathbb{N}$. Using the definition of the norm, one can recursively construct pairwise disjoint sets $F_{n}^{\prime} \in[\mathbb{N}]^{<\omega}$ such that there is $k_{n} \in \mathbb{N}$ satisfying $\sum_{i \in F_{n}^{\prime}}\left|m_{k_{n}, i}\right|>\varepsilon$ for each $n \in \mathbb{N}$. Grouping the elements $i \in F_{n}^{\prime}$ in four groups depending on the quadrant of the complex plane in which $m_{k_{n}, i}$ lies, we can choose subsets $F_{n} \subseteq F_{n}^{\prime}$ such that $\left|\sum_{i \in F_{n}} m_{k_{n}, i}\right|>\varepsilon /(4 \sqrt{2})$. The same $k_{n}$ cannot repeat itself more than $4 \sqrt{2}\|M\| / \varepsilon$ times, so we may assume that the sequence $\left(k_{n}\right)$ is strictly increasing. Thus the condition of the lemma fails.

Definition 25. Suppose that $M=\left(m_{k, n}\right)_{k, n \in \mathbb{N}} \in \mathbb{M}$. Then $D(M)=\left(m_{k, n}^{\prime}\right)_{k, n \in \mathbb{N}}$, where $m_{k, k}^{\prime}=m_{k, k}$ for all $k \in \mathbb{N}$ and $m_{k, n}^{\prime}=0$ whenever $k, n \in \mathbb{N}$ are distinct.

Lemma 26. Suppose that $M=\left(m_{k, n}\right)_{k, n \in \mathbb{N}} \in \mathbb{M}$ and $\lambda, \lambda^{\prime} \in \mathbb{K}$. Then:

(1) $M$ is compact if and only if $D(M)$ and $M-D(M)$ are compact.

(2) $D(M)$ is compact if and only if $\lim _{n \in \mathbb{N}} m_{n, n}=0$.

(3) If both $M-\lambda I$ and $M-\lambda^{\prime} I$ are compact, then $\lambda=\lambda^{\prime}$.

Proof. Items (1) and (2) follow directly from the definition of the compactness of a matrix. Under the hypothesis of $(3),(M-\lambda I)-\left(M-\lambda^{\prime} I\right)=\left(\lambda^{\prime}-\lambda\right) I$ is compact, and so $\lambda=\lambda^{\prime}$ by (2).

Lemma 27. Suppose that $M=\left(m_{k, n}\right)_{k, n \in \mathbb{N}} \in \mathbb{M}$ is not compact. Then there is an infinite and coinfinite $A \subseteq \mathbb{N}$ such that $\lim _{k \in \mathbb{N}}\left(M 1_{A}\right)(k)$ does not exist. If additionally $D(M)$ is a compact matrix, then there is an infinite and coinfinite $A \subseteq \mathbb{N}$ such that $\lim _{k \in \mathbb{N} \backslash A}\left(M 1_{A}\right)(k)$ does not exist. 
Proof. First we will note that if the columns of $M$, that is, the sequences $M 1_{\{n\}}$, do not all converge, then we get the stronger conclusion of the second part of the lemma. Indeed, suppose that $M 1_{\{n\}}$ does not converge for some $n \in \mathbb{N}$. Let $A \subseteq \mathbb{N}$ be infinite and coinfinite such that $\lim _{k \in \mathbb{N} \backslash A}\left(M 1_{\{n\}}\right)(k)$ does not exist and $n \notin A$. If $\lim _{k \in \mathbb{N} \backslash A}\left(M 1_{A}\right)(k)$ does not exist, we are done. Otherwise the set $A^{\prime}=A \cup\{n\}$ has the required property because if $\lim _{k \in \mathbb{N} \backslash A}\left(M 1_{A}\right)(k)$ and $\lim _{k \in \mathbb{N} \backslash A^{\prime}}\left(M 1_{A^{\prime}}\right)(k)$ both exist, then so does

$$
\lim _{k \in \mathbb{N} \backslash A^{\prime}}\left(M 1_{\{n\}}\right)(k)=\lim _{k \in \mathbb{N} \backslash A^{\prime}}\left(M 1_{A^{\prime}}\right)(k)-\lim _{k \in \mathbb{N} \backslash A^{\prime}}\left(M 1_{A}\right)(k),
$$

which is a contradiction.

Next we will prove the lemma under the additional hypothesis that each column of $M$ converges to 0 . By Lemma 24 and the hypothesis of the lemma, we can find pairwise disjoint sets $\left(F_{n}\right)_{n \in \mathbb{N}} \subseteq[\mathbb{N}]^{<\omega}$, a strictly increasing sequence $\left(k_{n}\right)_{n \in \mathbb{N}} \subseteq \mathbb{N}$ and $\varepsilon>0$ such that $\left|\sum_{i \in F_{n}} m_{k_{n}, i}\right|>\varepsilon$ for every $n \in \mathbb{N}$. By recursion on $j \in \mathbb{N}$, we can construct a strictly increasing sequence $\left(n_{j}\right)_{j \in \mathbb{N}} \subseteq \mathbb{N}$ such that

(1) $\sum_{j^{\prime}<j} \sum_{i \in F_{n_{j^{\prime}}}}\left|m_{k_{n_{j}}, i}\right|<\frac{\varepsilon}{5}$ for every $j \in \mathbb{N}$, and

(2) $\left|\sum_{i \in F_{n_{j}}} m_{k_{n_{j^{\prime}}}, i}\right|<\frac{\varepsilon}{5 \cdot 2^{j}}$ for every $j^{\prime}<j$.

At stage $j$ of the recursion, use the fact that the columns of $M$ converge to 0 to satisfy (1), and use the fact that the sets $F_{n}$ are pairwise disjoint and the rows of $M$ are absolutely summable to satisfy (2). Let $A=\bigcup_{j \in \mathbb{N}} F_{n_{2 j}}$. By the choice of the sets $F_{n}$ and (1) and (2), we have $\left|\left(M 1_{A}\right)\left(k_{n_{2 j}}\right)\right|>\varepsilon-2 \varepsilon / 5$ and $\left|\left(M 1_{A}\right)\left(k_{n_{2 j+1}}\right)\right|<2 \varepsilon / 5$ for every $j \in \mathbb{N}$. Hence $M 1_{A}$ does not converge.

Now to prove the second part of the lemma under the additional hypothesis that each column converges to 0 , first note that the hypothesis of the second part means that $\lim _{k \in \mathbb{N}} m_{k, k}=0$ by Lemma 26 . In this case the recursive construction above can be modified to yield an infinite and coinfinite set $A \subseteq \mathbb{N}$ such that $\lim _{k \in \mathbb{N} \backslash A}\left(M 1_{A}\right)(k)$ does not exist by replacing the set $F_{n}$ with $F_{n}^{\prime}=F_{n} \backslash\left\{k_{n}\right\}$ for every $n \in \mathbb{N}$ in the recursion requirements (1) and (2) and adding two more conditions:

(3) $\left\{k_{n_{j^{\prime}}}: j^{\prime}<j\right\} \cap F_{n_{j}}^{\prime}=\emptyset$, and

(4) $k_{n_{j}} \notin \bigcup_{j^{\prime}<j} F_{n_{j^{\prime}}}^{\prime}$ for every $j \in \mathbb{N}$.

The possibility of satisfying the modified (1) and (2) follows from the hypothesis that $\lim _{k \in \mathbb{N}} m_{k, k}=0$, while (3) and (4) can be satisfied by choosing $n_{j}$ sufficiently large, using that the sets $F_{n}$ are pairwise disjoint. With such a modified construction we obtain $\left\{k_{n_{j}}: j \in \mathbb{N}\right\} \subseteq \mathbb{N} \backslash A$, and so $\lim _{k \in \mathbb{N} \backslash A}\left(M 1_{A}\right)(k)$ does not exist.

Finally let us prove the first part of the lemma in the general case when all columns of $M$ converge, but not necessarily to 0 . Let $\lambda_{n}=\lim _{k \in \mathbb{N}}\left(M 1_{\{n\}}\right)(k)$, and define $M^{\prime}=\left(m_{k, n}^{\prime}\right)_{k, n \in \mathbb{N}}$ by $m_{k, n}^{\prime}=\lambda_{n}$ for every $k, n \in \mathbb{N}$. Note that $M^{\prime} \in \mathbb{M}$ as $\sum_{n<i}\left|\lambda_{n}\right|$ can be approximated by $\sum_{n<i}\left|m_{k, n}\right| \leq\|M\|<\infty$ for any $i \in \mathbb{N}$ and sufficiently large $k \in \mathbb{N}$. Hence we have $M-M^{\prime} \in \mathbb{M}$ as well. Moreover, the summability of $\sum_{n \in \mathbb{N}}\left|\lambda_{n}\right|$ implies that $M^{\prime}$ is compact by the definition of compactness. We may now apply the previous case, where the columns of the matrix converge to 0 , to the matrix $M^{\prime \prime}=M-M^{\prime}$, which is noncompact since $M$ is noncompact and $M^{\prime}$ is compact. Hence there exists an infinite and coinfinite set $A \subseteq \mathbb{N}$ such that $\lim _{k \in \mathbb{N}}\left(M^{\prime \prime} 1_{A}\right)(k)$ does not exist. The definition of $M^{\prime}$ implies that $M^{\prime} f$ is a constant sequence, and thus convergent, for every $f \in \ell_{\infty}$. Therefore, using 
that $\left(M 1_{A}\right)(k)=\left(M^{\prime} 1_{A}\right)(k)+\left(M^{\prime \prime} 1_{A}\right)(k)$, we conclude that $\lim _{k \in \mathbb{N}}\left(M 1_{A}\right)(k)$ does not exist, as required. The argument for the second part of the lemma is analogous. One needs to note that $D\left(M^{\prime \prime}\right)$ is compact if $D(M)$ is. This follows from the above fact that $M^{\prime}$ is compact, and so $D\left(M^{\prime}\right)$ is compact by Lemma 26 .

Recall Definitions 13, 14 and 15.

Lemma 28. Suppose that $M \in \mathbb{M}$ and that $\mathcal{A} \subseteq[\mathbb{N}]^{\omega}$.

(1) (Admission) If $\mathcal{A}$ accepts $M$ and no element of $\mathcal{A}$ undermines $M$, then $\mathcal{A}$ admits $M$.

(2) (Monotonicity) If $\mathcal{A}$ accepts $M$, then $B$ accepts $M$ for every $B \in[\mathbb{N}]^{\omega}$ which is included in a finite union of elements of $\mathcal{A}$.

(3) (Decidability) If $A \in[\mathbb{N}]^{\omega}$ does not accept $M-\lambda I$ for any $\lambda \in \mathbb{K}$, then there is an infinite $B \subseteq A$ such that $B$ rejects $M$.

(4) (Amalgamation) If, for every $A, A^{\prime} \in \mathcal{A}$, there is $\lambda_{A, A^{\prime}} \in \mathbb{K}$ such that $A \cup A^{\prime}$ accepts $M-\lambda_{A, A^{\prime}} I$, then there is $\lambda \in \mathbb{K}$ such that $\mathcal{A}$ accepts $M-\lambda I$.

Proof. (Admission) Fix $A, A^{\prime} \in \mathcal{A}$. For every $f \in \ell_{\infty}$ and $j \in \mathbb{N}$, we have

$$
\left(M^{A \cup A^{\prime}}-M_{j}^{A \cup A^{\prime}}\right) f=\sum_{n \leq j} f(n) M^{A \cup A^{\prime}} 1_{\{n\}},
$$

which belongs to $c_{0}$ because the hypothesis that $A$ and $A^{\prime}$ do not undermine $M$ implies that $\lim _{k \in A \cup A^{\prime}}\left(M 1_{\{n\}}\right)(k)=0$ for every $n \in \mathbb{N}$. Since

$$
M^{A \cup A^{\prime}} f=\left(M^{A \cup A^{\prime}}-M_{j}^{A \cup A^{\prime}}\right) f+M_{j}^{A \cup A^{\prime}} f,
$$

where $\left\|M_{j}^{A \cup A^{\prime}} f\right\| \leq\left\|M_{j}^{A \cup A^{\prime}}\right\|\|f\| \rightarrow 0$ as $j \rightarrow \infty$ by the compactness of $M^{A \cup A^{\prime}}$, we deduce that $M^{A \cup A^{\prime}} f$ can be approximated in the supremum norm by elements of $c_{0}$. This shows that $M^{A \cup A^{\prime}} f \in c_{0}$ because $c_{0}$ is a closed subspace of $\ell_{\infty}$. In particular, choosing $f=1_{A}$, we have

$$
0=\lim _{k \in A^{\prime}}\left(M^{A \cup A^{\prime}} 1_{A}\right)(k)=\lim _{k \in A^{\prime}}\left(M 1_{A}\right)(k) .
$$

Since $A$ and $A^{\prime}$ were arbitrary, this proves that $\mathcal{A}$ admits $M$.

(Monotonicity) Let $A_{1}, \ldots, A_{l} \in \mathcal{A}$. The compactness of each matrix $M^{A_{i} \cup A_{i^{\prime}}}$, which follows from the hypothesis that $\mathcal{A}$ accepts $M$, means that

$$
\lim _{j \in \mathbb{N}}\left(\sup _{k \in A_{i}} \sum_{n \in A_{i^{\prime}}, n>j}\left|m_{k, n}\right|\right)=0
$$

for every $1 \leq i, i^{\prime} \leq l$. But this implies that, for every $B \subseteq A_{1} \cup \cdots \cup A_{l}$, we have

$$
\lim _{j \in \mathbb{N}}\left(\sup _{k \in B} \sum_{n \in B, n>j}\left|m_{k, n}\right|\right)=0,
$$

which shows that $M^{B}$ is compact, as required.

(Decidability) First assume that $M^{A}-D\left(M^{A}\right)$ is not a compact matrix. Then, since $D\left(M^{A}-D\left(M^{A}\right)\right)$ is the zero matrix, we can apply a version of the second part of Lemma 27 with $\mathbb{N}$ replaced with $A$ to find an infinite and coinfinite set $B^{\prime} \subseteq A$ such that

$$
\lim _{k \in A \backslash B^{\prime}}\left(\left(M^{A}-D\left(M^{A}\right)\right) 1_{B^{\prime}}\right)(k)=\lim _{k \in A \backslash B^{\prime}}\left(M^{A} 1_{B^{\prime}}\right)(k)
$$

does not exist. If $\lim _{k \in A \backslash B^{\prime}}\left(M^{A} 1_{A \backslash B^{\prime}}\right)(k)$ does not exist, the conclusion follows by taking $B=A \backslash B^{\prime}$. Otherwise, as $1_{B^{\prime}}+1_{A \backslash B^{\prime}}=1_{A}$, we conclude that 
$\lim _{n \in A \backslash B^{\prime}}\left(M^{A} 1_{A}\right)(n)$ does not exist, which implies that $\lim _{n \in A}\left(M^{A} 1_{A}\right)(n)$ does not exist, i.e., $B=A$ rejects $M$.

Now suppose that $M^{A}-D\left(M^{A}\right)$ is compact. First we note that $\left(m_{k, k}\right)_{k \in A}$ does not converge. If it converged to $\lambda \in \mathbb{K}$, then $D\left(M^{A}\right)-\lambda I^{A}$ would be compact by Lemma 26(2) and so the identity

$$
(M-\lambda I)^{A}=\left(M^{A}-D\left(M^{A}\right)\right)+\left(D\left(M^{A}\right)-\lambda I^{A}\right)
$$

would contradict the hypothesis that $A$ does not accept $M-\lambda I$ for any $\lambda \in \mathbb{K}$.

Hence we can find two infinite, disjoint subsets $A_{1}$ and $A_{2}$ of $A$ such that $\left(m_{k, k}\right)_{k \in A_{1}}$ and $\left(m_{k, k}\right)_{k \in A_{2}}$ converge to distinct limits $\lambda_{1}$ and $\lambda_{2}$, respectively. Let $\varepsilon=\left|\lambda_{1}-\lambda_{2}\right|>0$. Since $M^{A}-D\left(M^{A}\right)$ is compact, there is $j \in \mathbb{N}$ such that $\left\|\left(M^{A}-D\left(M^{A}\right)\right)_{j}\right\|<\varepsilon / 3$. Let $B=\left(A_{1} \cup A_{2}\right) \backslash\{0, \ldots, j\}$. Then

$$
\left|\left(M 1_{B}\right)(k)-m_{k, k}\right|=\left|\left(\left(M^{A}-D\left(M^{A}\right)\right) 1_{B}\right)(k)\right|<\varepsilon / 3
$$

for every $k \in B$, so $\lim _{k \in B}\left(M 1_{B}\right)(k)$ does not exist, as required.

(Amalgamation) We need to find $\lambda \in \mathbb{K}$ such that $(M-\lambda I)^{A \cup A^{\prime}}$ is compact whenever $A, A^{\prime} \in \mathcal{A}$. For every pair $A, A^{\prime} \in \mathcal{A}$, we can choose $\lambda_{A, A^{\prime}} \in \mathbb{K}$ such that $\left(M-\lambda_{A, A^{\prime}} I\right)^{A \cup A^{\prime}}$ is compact by the hypothesis. Monotonicity implies that $\left(M-\lambda_{A, A^{\prime}} I\right)^{A}$ and $\left(M-\lambda_{A, A^{\prime \prime}} I\right)^{A}$ are compact for any $A, A^{\prime}, A^{\prime \prime} \in \mathcal{A}$, so $\lambda_{A, A^{\prime}}=\lambda_{A, A^{\prime \prime}}$ by Lemma 26(3). Hence $\lambda_{A, A^{\prime}}=\lambda_{A, A^{\prime \prime}}=\lambda_{A^{\prime \prime \prime}, A^{\prime \prime}}$ for any $A, A^{\prime}, A^{\prime \prime}, A^{\prime \prime \prime} \in \mathcal{A}$, so all of these numbers have the same value $\lambda$, as required.

Many of the results of this section can be interpreted in the language of bounded linear operators on Banach spaces rather than matrices. In fact this was our original motivation. As we already mentioned on page 12 , elements of $\mathbb{M}$ are exactly the matrices which represent bounded linear operators from $c_{0}$ into $\ell_{\infty}$, or the adjoints of operators in $\mathscr{B}\left(\ell_{1}\right)$, that is, the weak*-continuous operators on $\ell_{\infty}$. Here and below we use the dualities $c_{0}^{*}=\ell_{1}$ and $\ell_{1}^{*}=\ell_{\infty}$. Compact matrices define exactly compact operators from $c_{0}$ into $\ell_{\infty}$. Using the Schur property of $\ell_{1}$, one can easily show that an operator defined on $c_{0}$ is compact if an only if it is weakly compact.

The condition of Lemma 24 is the classical Dieudonné-Grothendieck characterization of weakly compact subsets of the dual of a $C(K)$-space (see, e.g., Section 5.3 of [1]). Lemma 27 and the Decidability of Lemma 28 are more specific versions of the result [14] which says that a non-weakly compact operator on a Grothendieck space $\left(\ell_{\infty}\right.$ in this case) cannot have separable range, so in particular its range cannot be contained in the space $c$ of convergent sequences. The Admission of Lemma 28 corresponds to the result which says that $T^{* *}\left[\mathcal{X}^{* *}\right] \subseteq \mathcal{X}$ for any weakly compact operator $T$ on a Banach space $\mathcal{X}$ (see, e.g., Appendix $\mathrm{G}$ of [1]). Here $\mathcal{X}=c_{0}$ and $\mathcal{X}^{* *}=\ell_{\infty}$, and one needs to note that applying the same matrix from $\mathbb{M}$ to elements of $c_{0}$ and elements of $\ell_{\infty}$ corresponds to the operator $T$ and its second adjoint $T^{* *}$, respectively. The Monotonicity of Lemma 28 corresponds to the fact that (weakly) compact operators form an ideal.

We opted for giving direct proofs in terms of $\mathbb{N} \times \mathbb{N}$ matrices because in any case Lemma 26 and the concrete conditions of Lemma 28 require us to work with the combinatorial structure of matrices. Also the above mentioned operator theoretic results require quite substantial abstract preparations before they can be applied in our setting. This is not necessary as the facts we have proved above are fairly elementary and, as we have seen, can essentially be deduced from elementary properties of the summability of infinite series. 


\section{Borel structure, matrices And Almost disjoint Families}

In this section we link $\mathbb{K}$-valued $\mathbb{N} \times \mathbb{N}$ matrices and almost disjoint families in $[\mathbb{N}]^{\omega}$ with the Borel structure of $2^{\mathbb{N}}$ and $2^{\mathbb{N}} \times 2^{\mathbb{N}}$. Here $2^{\mathbb{N}}$ is considered with the product topology, so in particular the sets of the form $[s]=\left\{p \in 2^{\mathbb{N}}: s \subseteq p\right\}$, where $s$ is a finite partial function from $\mathbb{N}$ into $2=\{0,1\}$, form a basis for the topology of $2^{\mathbb{N}}$ consisting of clopen sets.

Our aim is to transform the following cardinality dichotomy for Borel sets due to Alexandrov and Hausdorff:

Lemma 29 (A dichotomy for Borel sets). If $X \subseteq 2^{\mathbb{N}}$ is Borel, then either $X$ is countable or $X$ has cardinality $\mathfrak{c}$.

Proof. See 18.6 of [32].

into the dichotomy for acceptance and rejection (Lemma 35). The latter dichotomy will apply to Borel almost disjoint families, so first we need the following result:

Lemma 30. There is an uncountable, almost disjoint family $\mathcal{A} \subseteq[\mathbb{N}]^{\omega}$ such that $\left\{1_{A}: A \in \mathcal{A}\right\} \subseteq 2^{\mathbb{N}}$ is closed.

Proof. Let $2^{<\mathbb{N}}=\bigcup_{n \in \mathbb{N}} 2^{n}$, and let $\phi: \mathbb{N} \rightarrow 2^{<\mathbb{N}}$ be a bijection. Given $p \in 2^{\mathbb{N}}$, define $A_{p}=\{p \mid n: n \in \mathbb{N}\} \subseteq 2^{<\mathbb{N}}$, where $p \mid n \in 2^{n}$ is the restriction of $p$ to $\{0, \ldots, n-1\}$. It is clear that $\left\{A_{p}: p \in 2^{\mathbb{N}}\right\} \subseteq\left[2^{<\mathbb{N}}\right]^{\omega}$ is an almost disjoint family of cardinality .

Let $\mathcal{A}=\left\{\phi^{-1}\left[A_{p}\right]: p \in 2^{\mathbb{N}}\right\}$. We shall prove that $\left\{1_{A}: A \in \mathcal{A}\right\}$ is closed in $2^{\mathbb{N}}$ by showing that its complement is open. Let $1_{A} \in 2^{\mathbb{N}} \backslash\left\{1_{B}: B \in \mathcal{A}\right\}$. Then $\phi[A]$ is not of the form $A_{p}$ for any $p \in 2^{\mathbb{N}}$. There are three possible reasons for this:

(1) There are $s, t \in \phi[A]$ such that $s \nsubseteq t$ and $t \nsubseteq s$.

(2) There are $s, t \in 2^{<\mathbb{N}}$ such that $s \subseteq t$ and $t \in \phi[A]$, but $s \notin \phi[A]$.

(3) $A$ is finite, and so there is $n \in \mathbb{N}$ such that $\phi[A] \cap 2^{n}=\emptyset$.

All these cases define clopen neighbourhoods of $1_{A}$ disjoint from $\left\{1_{B}: B \in \mathcal{A}\right\}$, as required.

Now we step up the cardinality dichotomy for Borel subsets of $2^{\mathbb{N}}$ to its square $2^{\mathbb{N}} \times 2^{\mathbb{N}}$, using a result from [18] of van Engelen, Kunen and Miller.

Lemma 31. Suppose that $X \subseteq 2^{\mathbb{N}} \times 2^{\mathbb{N}}$ is a Borel set. Then one of the following conditions holds:

(1) either there is a countable $Y \subseteq 2^{\mathbb{N}}$ such that each point of $X$ has at least one of its coordinates in $Y$,

(2) or there is $Z \subseteq X$ of cardinality continuum such that for any distinct points $(p, q),\left(p^{\prime}, q^{\prime}\right) \in Z$, we have $\{p, q\} \cap\left\{p^{\prime}, q^{\prime}\right\}=\emptyset$.

Proof. As $2^{\mathbb{N}}$ can be embedded as a closed subset of $\mathbb{R}$, it is enough to prove the lemma for Borel subsets $X$ of $\mathbb{R}^{2}$.

First note that if there is a nonzero $a \in \mathbb{R}$ such that $X$ intersects the line $\ell$ given by $y=a x$ in an uncountable set, then the second alternative of the lemma is satisfied. Indeed, the intersection of $\ell$ with $X$ is Borel and uncountable, and so of cardinality continuum by Lemma 29. One can now construct the set $Z$ by an easy transfinite recursion of length continuum by choosing its elements from this intersection, using the fact that given a point $(p, q)$ on $\ell$, there may be at most two other points $\left(p^{\prime}, q^{\prime}\right)$ on $\ell$ (namely $(p / a, p)$ and $\left.(q, a q)\right)$ with $\{p, q\} \cap\left\{p^{\prime}, q^{\prime}\right\} \neq \emptyset$. 
The above observation implies the lemma in the case where $X$ is covered by countably many lines. Indeed, if there is a countable collection $\mathcal{L}$ of lines covering $X$, then $X=H \cup V \cup S$, where $H, V$ and $S$ are points on horizontal, vertical and sloping lines in $\mathcal{L}$, respectively. If $S$ is uncountable, we are in the second alternative of the lemma by the preceding observation. Otherwise $S$ is countable, so we can assume that $S=\emptyset$, as countably many points can be covered by countably many horizontal and/or vertical lines. Then the first alternative of the lemma is satisfied by the set $Y=\pi_{y}[H] \cup \pi_{x}[V]$, where $\pi_{x}$ and $\pi_{y}$ are the projections on the $x$-axis and $y$-axis, respectively.

Hence we are left with the case where $X$ cannot be covered by countably many lines. Then we can use a theorem of van Engelen, Kunen and Miller [18], which says that an analytic (in particular Borel) subset of the plane which cannot be covered by countably many lines contains a perfect subset $P$ (hence of size continuum) such that no three points in $P$ are collinear. One may now construct a set $Z \subseteq X$ such that the second alternative of the lemma is satisfied by choosing its elements from $P$ by an easy transfinite recursion of length continuum, using the fact that given a point $(p, q)$ in $P$, there are at most six other points $\left(p^{\prime}, q^{\prime}\right)$ in $P$ with $\{p, q\} \cap\left\{p^{\prime}, q^{\prime}\right\} \neq \emptyset$, namely at most one point on each of the lines $x=p$ and $y=q$, and at most two points on each of the lines $x=q$ and $y=p$.

Before we can establish our desired dichotomy for acceptance and rejection (Lemma 35), we need to verify that certain sets induced by $\mathbb{N} \times \mathbb{N}$ matrices are Borel. This is done in the following lemmas which culminate in Lemma 34.

Lemma 32. Suppose that $M=\left(m_{k, n}\right)_{k, n \in \mathbb{N}} \in \mathbb{M}$. Then

$$
C(M)=\left\{1_{A} \in 2^{\mathbb{N}}: M^{A} \text { is a compact matrix }\right\}
$$

is a Borel subset of $2^{\mathbb{N}}$.

Proof. Note that for every $i, j, k \in \mathbb{N}$, the set

$$
S_{i, j, k}(M)=\left\{1_{A} \in 2^{\mathbb{N}}: k \in A \Rightarrow \sum_{n \in A, n>j}\left|m_{k, n}\right| \leq \frac{1}{i+1}\right\}
$$

is closed in $2^{\mathbb{N}}$ because if $1_{A} \notin S_{i, j, k}$, then $\left[1_{A} \mid n\right] \cap S_{i, j, k}(M)=\emptyset$ for some $n \in \mathbb{N}$. By the definition of a compact matrix, we have

$$
C(M)=\bigcap_{i \in \mathbb{N}} \bigcup_{j \in \mathbb{N}} \bigcap_{k \in \mathbb{N}} S_{i, j, k}(M),
$$

so it follows that $C(M)$ is a Borel subset of $2^{\mathbb{N}}$, as required.

In the next two proofs, we use the notation $(n, i)$ for $n \in \mathbb{N}$ and $i \in\{0,1\}$ to denote the partial function which has domain $\{n\}$ and takes the value $i$ at $n$.

Lemma 33. Suppose that $M=\left(m_{k, n}\right)_{k, n \in \mathbb{N}} \in \mathbb{M}$. Then

$$
\operatorname{Conv}(M)=\left\{1_{A} \in 2^{\mathbb{N}}:\left(m_{n, n}\right)_{n \in A} \text { converges }\right\}
$$

is a Borel subset of $2^{\mathbb{N}}$.

Proof. For every $i, n, n^{\prime} \in \mathbb{N}$, the set

$$
\operatorname{Conv}_{i, n, n^{\prime}}(M)=\left\{1_{A} \in 2^{\mathbb{N}}: n, n^{\prime} \in A \Rightarrow\left|m_{n, n}-m_{n^{\prime}, n^{\prime}}\right|<\frac{1}{i+1}\right\}
$$


is clopen in $2^{\mathbb{N}}$ because it is equal to $2^{\mathbb{N}}$ if $\left|m_{n, n}-m_{n^{\prime}, n^{\prime}}\right|<1 /(i+1)$, and it is equal to $[(n, 0)] \cup\left[\left(n^{\prime}, 0\right)\right]$ otherwise. Hence the conclusion follows from the fact that

$$
\operatorname{Conv}(M)=\bigcap_{i \in \mathbb{N}} \bigcup_{j \in \mathbb{N}} \bigcap_{n, n^{\prime}>j} \operatorname{Conv}_{i, n, n^{\prime}}(M) .
$$

Lemma 34. Let $M \in \mathbb{M}$. Then

$$
E(M)=\left\{\left(1_{A}, 1_{A^{\prime}}\right) \in 2^{\mathbb{N}} \times 2^{\mathbb{N}}: A \cup A^{\prime} \text { accepts } M-\lambda I \text { for some } \lambda \in \mathbb{K}\right\}
$$

is a Borel subset of $2^{\mathbb{N}} \times 2^{\mathbb{N}}$.

Proof. By Lemma 26, $\left(1_{A}, 1_{A^{\prime}}\right) \in E(M)$ if and only if $1_{A \cup A^{\prime}} \in C(M-D(M))$ and $1_{A \cup A^{\prime}} \in \operatorname{Conv}(M)$. Hence

$$
E(M)=\phi^{-1}[C(M-D(M))] \cap \phi^{-1}[\operatorname{Conv}(M)],
$$

where $\phi: 2^{\mathbb{N}} \times 2^{\mathbb{N}} \rightarrow 2^{\mathbb{N}}$ is the function defined by $\phi\left(1_{A}, 1_{A^{\prime}}\right)=1_{A \cup A^{\prime}}$. To check the continuity of $\phi$, it is enough to note that, for any $n \in \mathbb{N}$,

$$
\phi^{-1}[[(n, 0)]]=[(n, 0)] \times[(n, 0)], \quad \phi^{-1}[[(n, 1)]]=2^{\mathbb{N}} \times[(n, 1)] \cup[(n, 1)] \times 2^{\mathbb{N}} .
$$

Therefore Lemmas 32 and 33 imply that $E(M)$ is a Borel subset of $2^{\mathbb{N}} \times 2^{\mathbb{N}}$.

Lemma 35 (A dichotomy for acceptance and rejection). Suppose that $M \in \mathbb{M}$ and $\mathcal{A} \subseteq[\mathbb{N}]^{\omega}$ is an uncountable, almost disjoint family such that $\left\{1_{A}: A \in \mathcal{A}\right\}$ is a Borel subset of $2^{\mathbb{N}}$. Then one of the following holds:

(1) either $\mathcal{A} \backslash \mathcal{A}^{\prime}$ accepts $M-\lambda I$ for some countable $\mathcal{A}^{\prime} \subseteq \mathcal{A}$ and $\lambda \in \mathbb{K}$,

(2) or there are pairwise disjoint sets $\left\{A_{\xi}, A_{\xi}^{\prime}\right\} \subseteq \mathcal{A}$ and an infinite subset $B_{\xi} \subseteq A_{\xi} \cup A_{\xi}^{\prime}$ such that $B_{\xi}$ rejects $M$ for every $\xi<\mathfrak{c}$.

Proof. Let $X(\mathcal{A})=\left\{1_{A}: A \in \mathcal{A}\right\}$. By Lemma 34 , the set

$$
X=(X(\mathcal{A}) \times X(\mathcal{A})) \backslash E(M)
$$

is Borel as the Cartesian product of two Borel sets is Borel, where we recall that

$$
E(M)=\left\{\left(1_{A}, 1_{A^{\prime}}\right) \in 2^{\mathbb{N}} \times 2^{\mathbb{N}}: A \cup A^{\prime} \text { accepts } M-\lambda I \text { for some } \lambda \in \mathbb{K}\right\} .
$$

We can therefore apply Lemma 31 to $X$.

The first alternative of Lemma 31 gives that there is a countable $\mathcal{A}^{\prime} \subseteq \mathcal{A}$ such that if $A, A^{\prime} \in \mathcal{A} \backslash \mathcal{A}^{\prime}$, then $\left(1_{A}, 1_{A^{\prime}}\right) \in E(M)$, that is, $A \cup A^{\prime}$ accepts $M-\lambda I$ for some $\lambda \in \mathbb{K}$. By the Amalgamation of Lemma 28, this means that there is a single $\lambda \in \mathbb{K}$ such that $\mathcal{A} \backslash \mathcal{A}^{\prime}$ accepts $M-\lambda I$.

The second alternative of Lemma 31 gives a set $Z \subseteq X$ of cardinality continuum such that $\left\{1_{A}, 1_{A^{\prime}}\right\} \cap\left\{1_{B}, 1_{B^{\prime}}\right\}=\emptyset$ for any distinct points $\left(1_{A}, 1_{A^{\prime}}\right),\left(1_{B}, 1_{B^{\prime}}\right) \in Z$. This yields a pairwise disjoint family $\left\{\left\{A_{\xi}, A_{\xi}^{\prime}\right\}: \xi<\mathfrak{c}\right\}$ with $A_{\xi}, A_{\xi}^{\prime} \in \mathcal{A}$ such that $A_{\xi} \cup A_{\xi}^{\prime}$ does not accept $M-\lambda I$ for any $\lambda \in \mathbb{K}$ and any $\xi<\mathfrak{c}$. By the Decidability of Lemma 28, this produces an infinite set $B_{\xi} \subseteq A_{\xi} \cup A_{\xi}^{\prime}$ such that $B_{\xi}$ rejects $M$.

\section{Consequences of Theorem 2, context and open questions}

In this section we shall establish some consequences of Theorem 2, first for the compact space $\alpha K_{\mathcal{A}}$ and then for the Banach algebra $\mathscr{B}\left(C_{0}\left(K_{\mathcal{A}}\right)\right)$ of all bounded linear operators on $C_{0}\left(K_{\mathcal{A}}\right)$. Moreover, we shall discuss the place of the Banach space $C_{0}\left(K_{\mathcal{A}}\right)$ among other known Banach spaces with few operators, and we shall present and motivate some open questions which arise naturally from our work. 


\subsection{Continuous self-maps of $\alpha K_{\mathcal{A}}$ when $C_{0}\left(K_{\mathcal{A}}\right)$ has few operators.}

Proposition 36. Suppose that $\mathcal{A} \subseteq[\mathbb{N}]^{\omega}$ is an uncountable, almost disjoint family such that all bounded linear operators on $C_{0}\left(K_{\mathcal{A}}\right)$ are of the form described in Theorem 2. Then, for every continuous map $\phi: \alpha K_{\mathcal{A}} \rightarrow \alpha K_{\mathcal{A}}$, either $\phi$ has countable range or the set of fixed points of $\phi$ is cocountable.

Proof. As $C_{0}\left(K_{\mathcal{A}}\right)$ is isomorphic to $C\left(\alpha K_{\mathcal{A}}\right)$, the hypothesis implies that every bounded linear operator on $C\left(\alpha K_{\mathcal{A}}\right)$ is the sum of a scalar multiple of the identity and an operator with separable range. Given a continuous map $\phi: \alpha K_{\mathcal{A}} \rightarrow \alpha K_{\mathcal{A}}$, we will apply this hypothesis to the composition operator $T_{\phi}: C\left(\alpha K_{\mathcal{A}}\right) \rightarrow C\left(\alpha K_{\mathcal{A}}\right)$ defined by $T_{\phi}(f)=f \circ \phi$ for $f \in C\left(\alpha K_{\mathcal{A}}\right)$.

We begin by showing that if $T_{\phi}$ has separable range, then $\phi$ has countable range. To this end, suppose that $\phi$ has uncountable range. Then there are distinct $B_{\xi} \in \mathcal{A}$ for $\xi<\omega_{1}$ such that $y_{B_{\xi}}=\phi\left(y_{A_{\xi}}\right)$ for some $A_{\xi} \in \mathcal{A}$. For $\xi<\eta<\omega_{1}$, we have

$$
\begin{aligned}
\left\|T_{\phi}\left(1_{U\left(B_{\xi}\right)}\right)-T_{\phi}\left(1_{U\left(B_{\eta}\right)}\right)\right\| & \geq\left|T_{\phi}\left(1_{U\left(B_{\xi}\right)}\right)\left(y_{A_{\xi}}\right)-T_{\phi}\left(1_{U\left(B_{\eta}\right)}\right)\left(y_{A_{\xi}}\right)\right| \\
& =\left|1_{U\left(B_{\xi}\right)}\left(\phi\left(y_{A_{\xi}}\right)\right)-1_{U\left(B_{\eta}\right)}\left(\phi\left(y_{A_{\xi}}\right)\right)\right| \\
& =\left|1_{U\left(B_{\xi}\right)}\left(y_{B_{\xi}}\right)-1_{U\left(B_{\eta}\right)}\left(y_{B_{\xi}}\right)\right|=1-0=1 .
\end{aligned}
$$

This shows that the range of $T_{\phi}$ is nonseparable, and the conclusion follows.

We are now ready to prove the dichotomy stated in the proposition. More precisely, we shall show that if the set of fixed points of $\phi$ is not cocountable, then $T_{\phi}$ has separable range, because it will then follow from the previous paragraph that $\phi$ has countable range. As explained in the first paragraph, the hypothesis of the proposition implies that there is a scalar $\lambda$ such that $T_{\phi}-\lambda \operatorname{Id}$ has separable range. Suppose that there are uncountably many points of $\alpha K_{\mathcal{A}}$ which are not fixed under $\phi$. Then we can choose distinct $B_{\xi} \in \mathcal{A}$ for $\xi<\omega_{1}$ such that $\phi\left(y_{B_{\xi}}\right) \neq y_{B_{\xi}}$. Set $z_{\xi}=\phi\left(y_{B_{\xi}}\right)$. Since the set $\left\{z_{\xi}: \xi<\omega_{1}\right\}$ is either countable or uncountable, by passing to a suitable uncountable subset of it, we may assume that

(1) either $z_{\xi}=z_{\eta}=z$ for all $\xi, \eta<\omega_{1}$ and some $z \in \alpha K_{\mathcal{A}}$, in which case we may further arrange that $z \in U\left(B_{\xi}\right)$ if and only if $z \in U\left(B_{\eta}\right)$ for any $\xi, \eta<\omega_{1}$

(2) or by a transfinite recursive construction, there are distinct $A_{\xi} \in \mathcal{A}$ for $\xi<\omega_{1}$ such that $z_{\xi}=y_{A_{\xi}}$ and $A_{\xi} \neq B_{\eta}$ for any $\xi, \eta<\omega_{1}$.

Then, for $\xi<\eta<\omega_{1}$, we have

$$
\begin{aligned}
\left\|\left(T_{\phi}-\lambda \mathrm{Id}\right)\left(1_{U\left(B_{\xi}\right)}\right)-\left(T_{\phi}-\lambda \mathrm{Id}\right)\left(1_{U\left(B_{\eta}\right)}\right)\right\| & \geq \\
\left|\left(T_{\phi}-\lambda \mathrm{Id}\right)\left(1_{U\left(B_{\xi}\right)}\right)\left(y_{B_{\xi}}\right)-\left(T_{\phi}-\lambda \mathrm{Id}\right)\left(1_{U\left(B_{\eta}\right)}\right)\left(y_{B_{\xi}}\right)\right| & = \\
\left.\mid 1_{U\left(B_{\xi}\right)}\left(z_{\xi}\right)-\lambda-1_{U\left(B_{\eta}\right)}\left(z_{\xi}\right)\right) \mid & =|\lambda|
\end{aligned}
$$

because in case (1) $1_{U\left(B_{\xi}\right)}\left(z_{\xi}\right)=1_{U\left(B_{\eta}\right)}\left(z_{\xi}\right)$, while in case (2) $z_{\xi}=y_{A_{\xi}} \notin\left\{y_{B_{\xi}}, y_{B_{\eta}}\right\}$. Since $\lambda$ was chosen such that $T_{\phi}-\lambda$ Id has separable range, we must have $\lambda=0$, so $T_{\phi}$ has separable range, as desired.

6.2. Automatic continuity of homomorphisms from $\mathscr{B}\left(C_{0}\left(K_{\mathcal{A}}\right)\right)$. Ever since Johnson [26] proved that every algebra homomorphism from $\mathscr{B}(\mathcal{X})$ into a Banach algebra is continuous whenever the Banach space $\mathcal{X}$ is isomorphic to its square $\mathcal{X} \oplus \mathcal{X}$, it has been a natural question whether this is also true for infinite-dimensional Banach spaces $\mathcal{X}$ which are not isomorphic to their squares. The first examples of 
Banach spaces with that property were not found until 1960, just a few years before Johnson's paper. They were:

- the quasi-reflexive Banach space $\mathcal{J}$ constructed by James [25], as shown by Bessaga and Pełczyński [8], and

- the space of continuous functions $C\left(\left[0, \omega_{1}\right]\right)$, as shown by Semadeni [53], where $\left[0, \omega_{1}\right]$ denotes the compact Hausdorff space consisting of all ordinals not exceeding $\omega_{1}$, endowed with the order topology.

Willis [61] and Ogden [46] have proved that all homomorphisms from $\mathscr{B}(\mathcal{X})$ into a Banach algebra are indeed continuous for these two spaces. However, this conclusion does not extend to all Banach spaces because Read [50] has constructed a Banach space $\mathcal{X}_{\mathrm{R}}$ such that $\mathscr{B}\left(\mathcal{X}_{\mathrm{R}}\right)$ admits a discontinuous derivation, and hence a discontinuous homomorphism into a Banach algebra. Dales, Loy and Willis [13] have subsequently given an example of a Banach space $\mathcal{X}_{\text {DLW }}$ such that all derivations from $\mathscr{B}\left(\mathcal{X}_{\mathrm{DLW}}\right)$ are continuous, but, assuming the Continuum Hypothesis $(\mathrm{CH})$, there is a discontinuous homomorphism from $\mathscr{B}\left(\mathcal{X}_{\mathrm{DLW}}\right)$ into a Banach algebra.

When $K$ is an infinite metrizable compact space, or in other words when $C(K)$ is an infinite-dimensional separable Banach space, all homomorphisms from $\mathscr{B}(C(K))$ into a Banach algebra are continuous by Johnson's original result because the isomorphic classification of separable $C(K)$-spaces due to Milutin, Bessaga and Pelczyński implies that $C(K)$ is isomorphic to its square.

On the other hand, the Banach space $C_{0}\left(K_{\mathcal{A}}\right)$ obtained in [34] or Theorem 2 is clearly not isomorphic to its square, so the question arises whether every homomorphism from $\mathscr{B}\left(C_{0}\left(K_{\mathcal{A}}\right)\right)$ into a Banach algebra is continuous. The aim of this subsection is to show that this is indeed true. Our proof uses standard techniques, following closely the approach of Willis [61], which is based on the following notion.

Definition 37. A bounded linear operator $T$ on a Banach space $\mathcal{X}$ is compressible if, for some integer $n \geq 1$, there is a sequence of bounded linear projections $\left(Q_{j}\right)$ on the direct $\operatorname{sum} \mathcal{X}^{n}$ of $n$ copies of $\mathcal{X}$ such that:

(i) $Q_{j} Q_{k}=0$ whenever $j, k \in \mathbb{N}$ are distinct, and

(ii) $T$ factors through $Q_{j}$ for every $j \in \mathbb{N}$.

Before we state our central result, which will have the desired conclusion as an immediate consequence, recall that $\mathscr{X}\left(C_{0}\left(K_{\mathcal{A}}\right)\right)$ denotes the ideal of $\mathscr{B}\left(C_{0}\left(K_{\mathcal{A}}\right)\right)$ consisting of all operators with separable range. By Lemma 22, an operator belongs to this ideal if and only if it factors through $c_{0}$.

Lemma 38. Let $\mathcal{A} \subseteq[\mathbb{N}]^{\omega}$ be an almost disjoint family. Then:

(1) Every operator in the ideal $\mathscr{X}\left(C_{0}\left(K_{\mathcal{A}}\right)\right)$ is compressible.

(2) Null sequences in $\mathscr{X}\left(C_{0}\left(K_{\mathcal{A}}\right)\right)$ factor in the following precise sense: whenever $\left(T_{j}\right)$ is a sequence in $\mathscr{X}\left(C_{0}\left(K_{\mathcal{A}}\right)\right)$ such that $\left\|T_{j}\right\| \rightarrow 0$ as $j \rightarrow \infty$, there are $S \in \mathscr{X}\left(C_{0}\left(K_{\mathcal{A}}\right)\right)$ and a sequence $\left(R_{j}\right)$ in $\mathscr{X}\left(C_{0}\left(K_{\mathcal{A}}\right)\right)$ such that $T_{j}=S R_{j}$ for every $j \in \mathbb{N}$ and $\left\|R_{j}\right\| \rightarrow 0$ as $j \rightarrow \infty$.

(3) Every homomorphism from $\mathscr{X}\left(C_{0}\left(K_{\mathcal{A}}\right)\right)$ into a Banach algebra is continuous.

Proof. Since $C_{0}\left(K_{\mathcal{A}}\right)$ contains a complemented copy of $c_{0}$, we can find bounded linear operators $U: C_{0}\left(K_{\mathcal{A}}\right) \rightarrow c_{0}$ and $V: c_{0} \rightarrow C_{0}\left(K_{\mathcal{A}}\right)$ such that $U V=\operatorname{Id}_{c_{0}}$.

(1). Suppose that $T \in \mathscr{X}\left(C_{0}\left(K_{\mathcal{A}}\right)\right)$. We shall show that Definition 37 is satisfied for $n=1$. The fact that $c_{0}$ is isomorphic to the $c_{0}$-direct sum of countably many 
copies of itself means that we can find a sequence $\left(P_{j}\right)$ of projections in $\mathscr{B}\left(c_{0}\right)$ such that $P_{j} P_{k}=0$ whenever $j, k \in \mathbb{N}$ are distinct and $P_{j}\left[c_{0}\right] \sim c_{0}$ for every $j \in \mathbb{N}$. Then the operators $Q_{j}=V P_{j} U \in \mathscr{B}\left(C_{0}\left(K_{\mathcal{A}}\right)\right)$ for $j \in \mathbb{N}$ are projections which satisfy part (i) of Definition 37 because $U V=\operatorname{Id}_{c_{0}}$, and this fact also implies that $Q_{j}\left[C_{0}\left(K_{\mathcal{A}}\right)\right] \sim P_{j}\left[c_{0}\right] \sim c_{0}$ for every $j \in \mathbb{N}$.

To verify part (ii) of Definition 37, fix $j \in \mathbb{N}$. By Lemma 22 , we can find a closed subspace $\mathcal{Y}$ of $C_{0}\left(K_{\mathcal{A}}\right)$ such that $T\left[C_{0}\left(K_{\mathcal{A}}\right)\right] \subseteq \mathcal{Y}$ and $\mathcal{Y} \sim c_{0}$, and hence there is an isomorphism $W: \mathcal{Y} \rightarrow Q_{j}\left[C_{0}\left(K_{\mathcal{A}}\right)\right]$. Define $R=J W T \in \mathscr{B}\left(C_{0}\left(K_{\mathcal{A}}\right)\right)$ and $S=$ $J^{\prime} W^{-1} Q_{j} \in \mathscr{B}\left(C_{0}\left(K_{\mathcal{A}}\right)\right)$, where $J: Q_{j}\left[C_{0}\left(K_{\mathcal{A}}\right)\right] \rightarrow C_{0}\left(K_{\mathcal{A}}\right)$ and $J^{\prime}: \mathcal{Y} \rightarrow C_{0}\left(K_{\mathcal{A}}\right)$ are the inclusions. Then it is easy to see that $T=S Q_{j} R$, as desired. (Alternatively, this can be shown using Proposition 1 in [61].)

(2). Let $\left(T_{j}\right)$ be a null sequence in $\mathscr{X}\left(C_{0}\left(K_{\mathcal{A}}\right)\right)$. Since $\bigcup_{j \in \mathbb{N}} T_{j}\left[C_{0}\left(K_{\mathcal{A}}\right)\right]$ spans a separable subspace of $C_{0}\left(K_{\mathcal{A}}\right)$, Lemma 22 implies that there is a closed subspace $\mathcal{Y}$ of $C_{0}\left(K_{\mathcal{A}}\right)$ such that $\bigcup_{j \in \mathbb{N}} T_{j}\left[C_{0}\left(K_{\mathcal{A}}\right)\right] \subseteq \mathcal{Y}$ and $\mathcal{Y} \sim c_{0}$. Let $W: \mathcal{Y} \rightarrow c_{0}$ be an isomorphism, and define $R_{j}=V W T_{j} \in \mathscr{X}\left(C_{0}\left(K_{\mathcal{A}}\right)\right)$ for $j \in \mathbb{N}$ and $S=$ $J W^{-1} U \in \mathscr{X}\left(C_{0}\left(K_{\mathcal{A}}\right)\right)$, where $J: \mathcal{Y} \rightarrow C_{0}\left(K_{\mathcal{A}}\right)$ is the inclusion and we recall that the operators $U$ and $V$ were chosen at the beginning of the proof. Then $S R_{j}=T_{j}$ for every $j \in \mathbb{N}$ because $U V=\operatorname{Id}_{c_{0}}$, and $\left\|R_{j}\right\| \leq\|V\|\|W\|\left\|T_{j}\right\| \rightarrow 0$ as $j \rightarrow \infty$, as desired.

(3). Let $\theta: \mathscr{X}\left(C_{0}\left(K_{\mathcal{A}}\right)\right) \rightarrow \mathscr{C}$ be a homomorphism into a Banach algebra $\mathscr{C}$, and consider the associated continuity ideal, which is defined by

$$
\begin{aligned}
\mathscr{I}=\left\{S \in \mathscr{X}\left(C_{0}\left(K_{\mathcal{A}}\right)\right):\right. & \text { the maps } T \mapsto \theta(S T), \mathscr{X}\left(C_{0}\left(K_{\mathcal{A}}\right)\right) \rightarrow \mathscr{C}, \\
& \text { and } \left.T \mapsto \theta(T S), \mathscr{X}\left(C_{0}\left(K_{\mathcal{A}}\right)\right) \rightarrow \mathscr{C}, \text { are continuous }\right\} .
\end{aligned}
$$

Suppose that $S \in \mathscr{X}\left(C_{0}\left(K_{\mathcal{A}}\right)\right)$. Applying part (2) to the trivial null sequence $(S, 0,0, \ldots)$ in $\mathscr{X}\left(C_{0}\left(K_{\mathcal{A}}\right)\right)$, we see that $S$ can be written as $S=R R^{\prime}$ for some $R, R^{\prime} \in \mathscr{X}\left(C_{0}\left(K_{\mathcal{A}}\right)\right)$. The same argument applied to $R^{\prime}$ instead of $S$ shows that $R^{\prime}=T T^{\prime}$ for some $T, T^{\prime} \in \mathscr{X}\left(C_{0}\left(K_{\mathcal{A}}\right)\right)$. By Proposition 7 of [61], we have $R T T^{\prime} \in \mathscr{I}$ whenever $T \in \mathscr{B}\left(C_{0}\left(K_{\mathcal{A}}\right)\right)$ is compressible and $R, T^{\prime} \in \mathscr{X}\left(C_{0}\left(K_{\mathcal{A}}\right)\right)$. Since every operator $T$ in $\mathscr{X}\left(C_{0}\left(K_{\mathcal{A}}\right)\right)$ is compressible by part (1), we conclude that $S=R T T^{\prime} \in \mathscr{I}$.

We can now show that $\theta$ is continuous. Suppose that $\left(T_{j}\right)$ is a null sequence in $\mathscr{X}\left(C_{0}\left(K_{\mathcal{A}}\right)\right)$. By part $(2)$, we can find $S \in \mathscr{X}\left(C_{0}\left(K_{\mathcal{A}}\right)\right)$ and a null sequence $\left(R_{j}\right)$ in $\mathscr{X}\left(C_{0}\left(K_{\mathcal{A}}\right)\right)$ such that $T_{j}=S R_{j}$ for every $j \in \mathbb{N}$. As shown above, we have $S \in \mathscr{I}$, so the map $R \mapsto \theta(S R), \mathscr{X}\left(C_{0}\left(K_{\mathcal{A}}\right)\right) \rightarrow \mathscr{C}$, is continuous, and therefore

$$
\theta\left(T_{j}\right)=\theta\left(S R_{j}\right) \rightarrow \theta(S \circ 0)=0 \quad \text { as } \quad j \rightarrow \infty .
$$

Corollary 39. Let $\mathcal{A} \subseteq\left[\mathbb{N}^{\omega}\right.$ be an almost disjoint family such that the ideal $\mathscr{X}\left(C_{0}\left(K_{\mathcal{A}}\right)\right)$ has finite codimension in $\mathscr{B}\left(C_{0}\left(K_{\mathcal{A}}\right)\right)$. Then every homomorphism from $\mathscr{B}\left(C_{0}\left(K_{\mathcal{A}}\right)\right)$ into a Banach algebra is continuous.

Proof. Let $\theta$ be a homomorphism from $\mathscr{B}\left(C_{0}\left(K_{\mathcal{A}}\right)\right)$ into a Banach algebra. Its restriction to $\mathscr{X}\left(C_{0}\left(K_{\mathcal{A}}\right)\right)$ is continuous by part (3) of Lemma 38 , and hence $\theta$ is continuous because $\mathscr{X}\left(C_{0}\left(K_{\mathcal{A}}\right)\right)$ is closed and has finite codimension in $\mathscr{B}\left(C_{0}\left(K_{\mathcal{A}}\right)\right)$.

Remark 40. None of the Banach spaces $\mathcal{X}$ such that $\mathscr{B}(\mathcal{X})$ admits a discontinuous homomorphism into a Banach algebra that we described at the beginning of this subsection have the form $C(K)$ for a compact Hausdorff space $K$. Hence, one 
may ask whether every homomorphism from $\mathscr{B}(C(K))$ into a Banach algebra is continuous whenever $K$ is a compact Hausdorff space. The following example shows that under $\mathrm{CH}$ this is not true. We do not know of such an example within ZFC.

The first named author has constructed a compact Hausdorff space $K$ such that every bounded linear operator on $C(K)$ is the sum of a multiplication operator and a weakly compact operator (see Theorem 6.1 of [33]). Moreover, $K$ can be chosen without isolated points, in which case the quotient of $\mathscr{B}(C(K))$ by the ideal of weakly compact operators is isomorphic to $C(K)$ as a Banach algebra by Theorem 6.5(i) of [12]. Hence there is a surjective, continuous homomorphism $\pi: \mathscr{B}(C(K)) \rightarrow C(K)$. A famous result of Dales [10] and Esterle [20] states that under $\mathrm{CH}, C(K)$ admits a discontinuous homomorphism $\theta$ into a Banach algebra whenever $K$ is an infinite compact Hausdorff space. Since $\pi$ is surjective, it follows that the composition $\theta \circ \pi$ is a discontinuous homomorphism from $\mathscr{B}(C(K))$ into a Banach algebra.

Note that the original construction in [33] of the space $K$ also assumed $\mathrm{CH}$, but Plebanek [49] has subsequently modified it to remove that assumption. A survey of this body of work is given in [35].

6.3. Banach spaces with few operators and characters on $\mathscr{B}(\mathcal{X})$. The question "Which kinds of bounded linear operators must exist on a Banach space?" has a long history, culminating in the spectacular resolution of the "scalar-plus-compact" problem a decade ago by Argyros and Haydon [4], who produced a Banach space on which every bounded linear operator is a compact perturbation of a scalar multiple of the identity. A key ingredient, and the seminal result in this line of research, is the construction by Gowers and Maurey [22] of a Banach space on which every bounded linear operator is a strictly singular perturbation of a scalar multiple of the identity, where we recall that a bounded linear operator is strictly singular if no restriction of it to an infinite-dimensional subspace is an isomorphism onto its range. We refer to [43] for a much more detailed survey of the work of Gowers and Maurey and its context.

Perhaps the earliest construction of a Banach space with "few operators" is due to Shelah [55], who found a nonseparable Banach space on which every bounded linear operator is the sum of a scalar multiple of the identity and an operator with separable range. Shelah's original example relied on an additional set-theoretic axiom, $\diamond$, but this assumption was later removed by Shelah and Steprāns [56]. Wark $[58,59]$ has taken this line of research further by producing a reflexive and, much more recently, a uniformly convex space with the above property. Note that the space $C_{0}\left(K_{\mathcal{A}}\right)$ from [34] or Theorem 2 is another instance of a nonseparable Banach space with the property that every bounded linear operator on it is the sum of a scalar multiple of the identity and an operator with separable range.

The above-mentioned Banach spaces of Argyros and Haydon, Gowers and Maurey, Shelah, Steprāns and Wark all have very complex definitions. By contrast, Banach spaces of the form $C(K)$ for a compact Hausdorff space $K$ are among the simplest Banach spaces that one can define, so a natural question is: To what extent can a $C(K)$-space have few operators in any similar sense?

To make this question more precise, we observe that a common feature of all the different variants of Banach spaces $\mathcal{X}$ with few operators that we have described above is that the Banach algebra $\mathscr{B}(\mathcal{X})$ has a maximal ideal of codimension one, and therefore the quotient map induces a character on $\mathscr{B}(\mathcal{X})$, that is, a nonzero 
linear functional $\varphi: \mathscr{B}(\mathcal{X}) \rightarrow \mathbb{K}$ which is multiplicative in the sense that $\varphi(T S)=$ $\varphi(T) \varphi(S)$ for all $S, T \in \mathscr{B}(\mathcal{X})$. Clearly, the kernel of a character is an ideal of codimension one, thus a maximal ideal, and it is closed.

Characters on the Banach algebra $\mathscr{B}(\mathcal{X})$ are not common due to the following result (see, e.g., Theorem 2.5.11 of [11]):

Theorem 41. Suppose that $\mathcal{X}$ is a Banach space which is isomorphic to its square $\mathcal{X} \oplus \mathcal{X}$. Then $\mathscr{B}(\mathcal{X})$ has no closed two-sided ideals of finite codimension, and hence there are no characters on $\mathscr{B}(\mathcal{X})$.

Recall from Subsection 6.2 that James' quasi-reflexive space $\mathcal{J}$ and $C\left(\left[0, \omega_{1}\right]\right)$ were the first infinite-dimensional Banach spaces shown not to be isomorphic to their squares. It turns out that both $\mathscr{B}(\mathcal{J})$ and $\mathscr{B}\left(C\left(\left[0, \omega_{1}\right]\right)\right)$ admit a character. In 1969, Berkson and Porta [7] identified the character on $\mathscr{B}(\mathcal{J})$ explicitly. A year later Edelstein and Mityagin [17] described both characters. However, the character on $\mathscr{B}\left(C\left(\left[0, \omega_{1}\right]\right)\right)$ can already be found implicitly in Semadeni's paper, as explained in Proposition 2.5 of [31], as well as in a subsequent paper of Alspach and Benyamini [2]. Loy and Willis [40] have studied these two characters in much greater detail. In both cases, $\mathscr{B}(\mathcal{X})$ admits only one character, as shown in [38] and [29], respectively.

The above-mentioned result of Argyros and Haydon shows the existence of a Banach space $\mathcal{X}_{\mathrm{AH}}$ such that $\mathscr{B}\left(\mathcal{X}_{\mathrm{AH}}\right)$ admits a character with the smallest possible kernel. (Here we are using the fact that the Banach space they constructed has a Schauder basis, so the ideal of finite-rank operators, which is contained in every nonzero two-sided ideal of $\mathscr{B}(\mathcal{X})$, is dense in the ideal of compact operators.) This result, as well as that of Gowers and Maurey, has no direct analogue for $C(K)$ spaces, as the following proposition shows.

Proposition 42. Suppose that $K$ is an infinite compact Hausdorff space. Then the ideal of strictly singular operators has infinite codimension in $\mathscr{B}(C(K))$.

Proof. We split in two cases. If the set $K^{\prime}$ of nonisolated points in $K$ is finite, then

$$
C(K) \sim \ell_{\infty}\left(K^{\prime}\right) \oplus c_{0}\left(K \backslash K^{\prime}\right) \sim c_{0}\left(K_{d}\right),
$$

where $K_{d}$ denotes the set $K$ endowed with the discrete topology. Hence the conclusion follows from the fact that the result is true for $c_{0}$.

Otherwise $K^{\prime}$ is infinite, and we can recursively construct a sequence $\left(x_{n}\right)$ in $K^{\prime}$ and disjoint open sets $\left(U_{n}\right)$ such that $x_{n} \in U_{n}$ for every $n \in \mathbb{N}$. Choose a function $f_{n} \in C(K)$ such that $f_{n}\left(x_{n}\right)=1$ and $\operatorname{supp} f_{n} \subseteq U_{n}$ for every $n \in \mathbb{N}$. We shall now complete the proof by showing that the multiplication operators $M_{f_{n}}: C(K) \rightarrow C(K)$ defined by $M_{f_{n}}(g)=f_{n} g$ are linearly independent over the ideal of strictly singular operators. To this end, suppose that $S=\sum_{j=1}^{n} \lambda_{j} M_{f_{j}}$ is a strictly singular operator for some $n \in \mathbb{N}$ and some scalars $\lambda_{1}, \ldots, \lambda_{n}$. We observe that $S$ is equal to the multiplication operator $M_{f}$, where $f=\sum_{j=1}^{n} \lambda_{j} f_{j} \in C(K)$. The strict singularity of $S=M_{f}$ means that the set $\{x \in K:|f(x)| \geq \varepsilon\}$ is finite for every $\varepsilon>0$. In particular $f$ vanishes at every nonisolated point of $K$, so $0=f\left(x_{j}\right)=\lambda_{j}$ for each $j \in\{1, \ldots, n\}$, as required.

Remark 43. (1) Even though we do not need it here, for context it is perhaps worth remarking that Pełczyński [47] has shown that for a compact Hausdorff space $K$, a bounded linear operator from $C(K)$ into a Banach space is strictly singular if and only if it is weakly compact. 
(2) As mentioned in Remark 40, the first named author [33] has constructed a compact Hausdorff space $K$ such that every bounded linear operator on $C(K)$ is the sum of a multiplication operator and a weakly compact operator. Obviously, multiplication operators are bounded and linear on any $C(K)$-space, so this space may be viewed as a $C(K)$-space with "few operators" in a similar sense to Gowers and Maurey.

We observe that $\mathscr{B}(C(K))$ has no characters when $K$ is a metrizable compact space with at least two points because $\mathscr{B}(C(K))$ is isomorphic to the algebra of scalar $K \times K$ matrices if $K$ is finite, and $C(K)$ is isomorphic to its square otherwise. On the other hand, the map $\varphi$ given by $\varphi(\lambda \mathrm{Id}+S)=\lambda$ is an example of a character on $\mathscr{B}\left(C_{0}\left(K_{\mathcal{A}}\right)\right)$, where $\mathcal{A} \subseteq[\mathbb{N}]^{\omega}, \lambda \in \mathbb{K}$ and $S \in \mathscr{X}\left(C_{0}\left(K_{\mathcal{A}}\right)\right)$ are as in Theorem 2 .

We shall next show that the kernel of this character is as small as possible; see part (1) of Remark 45 below for the precise meaning of this statement. It involves two additional pieces of notation and terminology concerning a Banach space $\mathcal{X}$ : First, we write $\mathscr{G}_{c_{0}}(\mathcal{X})$ for the set of bounded linear operators on $\mathcal{X}$ which factor through $c_{0}$. This is a two-sided ideal of $\mathscr{B}(\mathcal{X})$, but not in general closed. It is always contained in the ideal $\mathscr{X}(\mathcal{X})$ of operators with separable range, which is closed. When $\mathcal{X}=C_{0}\left(K_{\mathcal{A}}\right)$ or $\mathcal{X}=C\left(\alpha K_{\mathcal{A}}\right)$ for an almost disjoint family $\mathcal{A} \subseteq[\mathbb{N}]^{\omega}$, we recall from Lemma 22 that these two ideals are equal, so in particular $\mathscr{G}_{c_{0}}(\mathcal{X})$ is closed in these cases. Second, $\mathcal{X}$ is a Grothendieck space if every weak*-convergent sequence in the dual space $\mathcal{X}^{*}$ converges weakly.

Proposition 44. Suppose that $K$ is an infinite compact Hausdorff space and that $\varphi$ is a character on $\mathscr{B}(C(K))$. Then $\mathscr{G}_{c_{0}}(C(K)) \subseteq \operatorname{ker} \varphi$.

If $C(K)$ is a Grothendieck space, then $\mathscr{X}(C(K))$, and thus $\mathscr{G}_{c_{0}}(C(K))$, is contained in the ideal of strictly singular operators, and so $\mathscr{X}(C(K))$ and $\mathscr{G}_{c_{0}}(C(K))$ have infinite codimension in $\mathscr{B}(C(K))$.

Proof. We split the proof in two parts, beginning with the case where $C(K)$ is a Grothendieck space. In this case Diestel [14] has shown that every operator $S \in \mathscr{X}(C(K))$ is weakly compact and therefore strictly singular by the abovementioned result of Pełczyński [47]. Hence $S \in \operatorname{ker} \varphi$ because Proposition 6.6 of [38] implies that, for every infinite-dimensional Banach space $\mathcal{X}$, every maximal two-sided ideal of $\mathscr{B}(\mathcal{X})$ contains the ideal of strictly singular operators. Now the main conclusion follows from the fact that $\mathscr{G}_{c_{0}}(C(K)) \subseteq \mathscr{X}(C(K))$, while the final statement is a consequence of Proposition 42.

It remains to prove that $\mathscr{G}_{c_{0}}(C(K)) \subseteq \operatorname{ker} \varphi$ when $C(K)$ is not a Grothendieck space. Schachermayer [52] has shown that in this case $C(K)$ contains a complemented copy of $c_{0}$, that is, $\mathscr{B}(C(K))$ contains a projection $P$ whose range is isomorphic to $c_{0}$. If $\varphi(P) \neq 0$, then we would obtain a character on $\mathscr{B}\left(c_{0}\right)$, which is impossible by Theorem 41 because $c_{0} \sim c_{0} \oplus c_{0}$. Hence $P \in \operatorname{ker} \varphi$. Now the conclusion follows because every operator $T \in \mathscr{G}_{c_{0}}(C(K))$ can be written as $T=S P R$ for some $R, S \in \mathscr{B}(C(K))$, so $\varphi(T)=\varphi(S) \varphi(P) \varphi(R)=0$.

Remark 45. (1) The fact that we have equality in the inclusion $\mathscr{G}_{c_{0}}(C(K)) \subseteq$ $\operatorname{ker} \varphi$ for $K=\alpha K_{\mathcal{A}}$, where $\mathcal{A} \subseteq[\mathbb{N}]^{\omega}$ is the almost disjoint family constructed in [34] or Theorem 2, justifies the claim that the character $\varphi$ on $\mathscr{B}\left(C\left(\alpha K_{\mathcal{A}}\right)\right)$ has the "smallest possible kernel" in these cases.

(2) Consider the class of Banach spaces of the form $C(K)$, where $K$ is a compact Hausdorff space whose $\omega_{1}$ 'st Cantor-Bendixson derivative is empty and 
$C(K)$ is a Lindelöf space in the weak topology. The first named author and Zieliński have shown that the question "Does this class contain a space on which every bounded linear operator is the sum of a scalar multiple of the identity and an operator with separable range?" is undecidable (see Corollary 3.3 and Theorem 4.1 of [36]). Note that this result does not apply to any space of the form $C\left(\alpha K_{\mathcal{A}}\right)$ for an almost disjoint family $\mathcal{A} \subseteq[\mathbb{N}]^{\omega}$ because these spaces are known not to be Lindelöf in their weak topology.

(3) With a little extra work, a stronger form of Proposition 42 can be proved, namely that the quotient of $\mathscr{B}(C(K))$ by the ideal $\mathscr{S}(C(K))$ of strictly singular operators is nonseparable for every infinite compact Hausdorff space $K$. We sketch the argument here.

If $C(K)$ contains a complemented copy of $c_{0}$, then the conclusion follows easily from the fact that the quotient of $\mathscr{B}\left(c_{0}\right)$ by the ideal of compact operators (which is equal to the ideal of strictly singular operators in this case) is nonseparable.

Otherwise the set $K^{\prime}$ of nonisolated points in $K$ is infinite, and $C(K)$ is a Grothendieck space by the above-mentioned result of Schachermayer [52]. Then $C\left(K^{\prime}\right)$ is also a Grothendieck space, for example because $C\left(K^{\prime}\right)$ is a quotient of $C(K)$ by taking restrictions of functions, and being a Grothendieck space passes to quotients. Therefore $C\left(K^{\prime}\right)$ is nonseparable, so the conclusion follows from the general fact (true whether or not $C(K)$ is a Grothendieck space) that $\left\|M_{f}-S\right\| \geq\left\|f \mid K^{\prime}\right\|$ for every $S \in \mathscr{S}(C(K))$ and $f \in C(K)$.

6.4. What is the quotient of $\mathscr{B}\left(C_{0}\left(K_{\mathcal{A}}\right)\right)$ by the ideal of operators with separable range? The question "Which unital Banach algebras are isomorphic to the quotient $\mathscr{B}(\mathcal{X}) / \mathscr{K}(\mathcal{X})$ for some Banach space $\mathcal{X}$ ?", where $\mathscr{K}(\mathcal{X})$ denotes the ideal of compact operators, has received considerable attention since the breakthrough of Argyros and Haydon [4], who expressed the scalar field in this way. Notably, Motakis, Puglisi and Zisimopoulou [44] have shown that the algebra $C(K)$ for every countably infinite compact Hausdorff space $K$ can also be realized in this form, while [57] and [30] contain similar conclusions for certain nonsemisimple finite-dimensional algebras.

We propose the following question as a counterpart of the above question in the present context, where we recall that for an almost disjoint family $\mathcal{A} \subseteq[\mathbb{N}]^{\omega}$, Lemma 22 implies that the ideal $\mathscr{X}\left(C_{0}\left(K_{\mathcal{A}}\right)\right)$ is equal to the ideal of operators which factor through $c_{0}$.

Question 46. Which unital Banach algebras of density at most $\mathfrak{c}$ can be isomorphic to the quotient algebra $\mathscr{B}\left(C_{0}\left(K_{\mathcal{A}}\right)\right) / \mathscr{X}\left(C_{0}\left(K_{\mathcal{A}}\right)\right)$ for some uncountable, almost disjoint family $\mathcal{A} \subseteq[\mathbb{N}]^{\omega}$ ?

The upper bound on the density of the quotient algebra follows from the fact that $\mathscr{B}\left(C_{0}\left(K_{\mathcal{A}}\right)\right)$ has density $\mathfrak{c}$.

Theorem 2 shows that the above quotient may be one-dimensional, that is, isomorphic to the scalar field $\mathbb{K}$. Further, for every integer $n \geq 2$, we can iterate our construction to realize the algebra of scalar $n \times n$ matrices as such a quotient by identifying $\mathbb{N}$ with the disjoint union of $n$ copies of itself and take a copy of the family $\mathcal{A}$ from Theorem 2 in each of these $n$ copies of $\mathbb{N}$. 
We can obtain more specific versions of Question 46 by seeking to realize only the Banach algebras belonging to a particular family, or simply particular Banach algebras, as such quotients. Important cases are:

(i) The family of unital, separable $C^{*}$-algebras.

(ii) The family of commutative, unital, separable $C^{*}$-algebras, that is, algebras of the form $C(K)$ for a compact metric space $K$.

(iii) The Banach algebra $\mathscr{B}(\mathcal{X})$ for some known infinite-dimensional Banach space $\mathcal{X}$ such as $\mathcal{X}=\ell_{2}$, or more generally $\mathcal{X}=\ell_{p}$ for some $1 \leq p<\infty$, $\mathcal{X}=c_{0}$ or $\mathcal{X}=C([0,1])$.

Note that if we could construct an almost disjoint family $\mathcal{A} \subseteq[\mathbb{N}]^{\omega}$ such that the quotient algebra $\mathscr{B}\left(C_{0}\left(K_{\mathcal{A}}\right)\right) / \mathscr{X}\left(C_{0}\left(K_{\mathcal{A}}\right)\right)$ is isomorphic to $C(K)$ for some infinite compact Hausdorff space $K$, then under $\mathrm{CH}$ we could construct a discontinuous homomorphism from $\mathscr{B}\left(C_{0}\left(K_{\mathcal{A}}\right)\right)$ into a Banach algebra as in Remark 40. This, together with Corollary 39, motivates the following question.

Question 47. Suppose that $\mathcal{A} \subseteq[\mathbb{N}]^{\omega}$ is an uncountable, almost disjoint family. Is every homomorphism from $\mathscr{B}\left(C_{0}\left(K_{\mathcal{A}}\right)\right)$ into a Banach algebra continuous?

Note that the corresponding question concerning homomorphisms from $C(K)$ for an infinite compact Hausdorff space $K$ is known to be undecidable in ZFC.

There is a considerable body of work which may help shed light on Question 46, as we shall now explain. Throughout, $\mathcal{A} \subseteq[\mathbb{N}]^{\omega}$ denotes an uncountable, almost disjoint family. We recall that such a family is called maximal if it is not properly contained in any other almost disjoint family in $[\mathbb{N}]^{\omega}$.

Let $C_{b}\left(K_{\mathcal{A}}\right)$ be the set of all scalar-valued, bounded continuous functions defined on $K_{\mathcal{A}}$. This is a unital, commutative $C^{*}$-algebra which is isomorphic to $C\left(\beta K_{\mathcal{A}}\right)$ for the Cech-Stone compactification $\beta K_{\mathcal{A}}$ of $K_{\mathcal{A}}$. Clearly $C_{b}\left(K_{\mathcal{A}}\right)$ contains $C_{0}\left(K_{\mathcal{A}}\right)$ as a closed ideal. In the language of $C^{*}$-algebras, the quotient $C_{b}\left(K_{\mathcal{A}}\right) / C_{0}\left(K_{\mathcal{A}}\right)$ is known as the corona algebra, while $C_{b}\left(K_{\mathcal{A}}\right)$ is the multiplier algebra of $C_{0}\left(K_{\mathcal{A}}\right)$.

Every function $g \in C_{b}\left(K_{\mathcal{A}}\right)$ induces a bounded linear operator $M_{g}$ on $C_{0}\left(K_{\mathcal{A}}\right)$ by multiplication, that is, $M_{g}$ is given by $M_{g}(f)=f g$. The map $g \mapsto M_{g}$ is an isometric algebra homomorphism of $C_{b}\left(K_{\mathcal{A}}\right)$ into $\mathscr{B}\left(C_{0}\left(K_{\mathcal{A}}\right)\right)$. We write $\mathscr{M}\left(C_{0}\left(K_{\mathcal{A}}\right)\right)$ for the range of this map, which is isometrically isomorphic to $C_{b}\left(K_{\mathcal{A}}\right)$ and thus to $C\left(\beta K_{\mathcal{A}}\right)$.

Lemma 48. Suppose that $\mathcal{A} \subseteq[\mathbb{N}]^{\omega}$ is a maximal almost disjoint family and that $g \in C_{b}\left(K_{\mathcal{A}}\right)$. Then $g \in C_{0}\left(K_{\mathcal{A}}\right)$ if and only if the multiplication operator $M_{g}$ has separable range. Consequently the corona algebra $C_{b}\left(K_{\mathcal{A}}\right) / C_{0}\left(K_{\mathcal{A}}\right)$ is isomorphic to

$$
\frac{\mathscr{M}\left(C_{0}\left(K_{\mathcal{A}}\right)\right)}{\mathscr{M}\left(C_{0}\left(K_{\mathcal{A}}\right)\right) \cap \mathscr{X}\left(C_{0}\left(K_{\mathcal{A}}\right)\right)} .
$$

Proof. Lemma 21 implies that the operator $M_{g}$ has separable range if and only if the set $s(g)=\left\{A \in \mathcal{A}: g\left(y_{A}\right) \neq 0\right\}$ is countable, so we seek to prove that the latter statement is equivalent to $g \in C_{0}\left(K_{\mathcal{A}}\right)$. One implication is clear, namely that $s(g)$ is countable for every $g \in C_{0}\left(K_{\mathcal{A}}\right)$.

Conversely, suppose that $g \in C_{b}\left(K_{\mathcal{A}}\right) \backslash C_{0}\left(K_{\mathcal{A}}\right)$. Then $L=\left\{x \in K_{\mathcal{A}}:|g(x)| \geq \varepsilon\right\}$ is noncompact for some $\varepsilon>0$. Define

$$
B=\left\{n \in \mathbb{N}:\left|g\left(x_{n}\right)\right| \geq \varepsilon / 2\right\} \quad \text { and } \quad \mathcal{A}^{\prime}=\left\{A \in \mathcal{A}: A \cap B \in[\mathbb{N}]^{\omega}\right\} .
$$


First, we observe that $B^{\prime}=B \backslash \bigcup \mathcal{A}^{\prime}$ is finite because otherwise $\mathcal{A} \cup\left\{B^{\prime}\right\}$ would be an almost disjoint family in $[\mathbb{N}]^{\omega}$ strictly larger than $\mathcal{A}$, contradicting the maximality of $\mathcal{A}$. Second, $\mathcal{A}^{\prime}$ cannot be finite because if it were, $L$ would be compact. Hence $\mathcal{B}=\left\{A \cap B: A \in \mathcal{A}^{\prime}\right\}$ is an infinite family of infinite, almost disjoint subsets of $B$. The maximality of $\mathcal{A}$ implies that $\mathcal{B}$ is maximal as well, so $\mathcal{B}$ must be uncountable because no countably infinite set contains a countably infinite maximal almost disjoint family. Hence $\mathcal{A}^{\prime}$ is also uncountable. The definition of $B$ implies that $g\left(y_{A}\right) \neq 0$ for every $A \in \mathcal{A}^{\prime}$, so $\mathcal{A}^{\prime} \subseteq s(g)$, proving that $s(g)$ is uncountable.

Remark 49. The conclusion of Lemma 48 fails if the almost disjoint family $\mathcal{A} \subseteq[\mathbb{N}]^{\omega}$ is not maximal. Indeed, in that case we can choose $B \in[\mathbb{N}]^{\omega}$ such that $A \cap B \in[\mathbb{N}]^{<\omega}$ for every $A \in \mathcal{A}$. Then $F=\left\{x_{n}: n \in B\right\}$ is a non-compact, clopen subset of $K_{\mathcal{A}}$, so $1_{F} \in C_{b}\left(K_{\mathcal{A}}\right) \backslash C_{0}\left(K_{\mathcal{A}}\right)$, but the corresponding multiplication operator $M_{1_{F}}$ has separable range.

It is well known that the corona algebra is isometrically isomorphic to $C\left(K_{\mathcal{A}}^{*}\right)$, where $K_{\mathcal{A}}^{*}=\beta K_{\mathcal{A}} \backslash K_{\mathcal{A}}$ is the Cech-Stone remainder of $K_{\mathcal{A}}$. In the case of a maximal almost disjoint family $\mathcal{A} \subseteq[\mathbb{N}]^{\omega}$, Lemma 48 implies that we can define an injective algebra homomorphism $\psi: C_{b}\left(K_{\mathcal{A}}\right) / C_{0}\left(K_{\mathcal{A}}\right) \rightarrow \mathscr{B}\left(C_{0}\left(K_{\mathcal{A}}\right)\right) / \mathscr{X}\left(C_{0}\left(K_{\mathcal{A}}\right)\right)$ of norm 1 by

$$
\psi\left(g+C_{0}\left(K_{\mathcal{A}}\right)\right)=M_{g}+\mathscr{X}\left(C_{0}\left(K_{\mathcal{A}}\right)\right)
$$

for every $C_{b}\left(K_{\mathcal{A}}\right)$. It can be shown that $\psi$ is isometric, and hence $C\left(K_{\mathcal{A}}^{*}\right)$ is isometrically isomorphic to a commutative subalgebra of $\mathscr{B}\left(C_{0}\left(K_{\mathcal{A}}\right)\right) / \mathscr{X}\left(C_{0}\left(K_{\mathcal{A}}\right)\right)$.

The Cech-Stone remainder of $K_{\mathcal{A}}$ for a maximal almost disjoint family $\mathcal{A} \subseteq[\mathbb{N}]^{\omega}$ has been well investigated in the literature. For example, Kulesza and Levy [37] have observed that the methods of [6] imply that under $\mathrm{CH}$, any separable compact Hausdorff space is homeomorphic to the Čech-Stone remainder of $K_{\mathcal{A}}$ for some maximal almost disjoint family $\mathcal{A} \subseteq[\mathbb{N}]^{\omega}$. On the other hand, Dow [16] has shown that it is consistent that the Cech-Stone remainder of $K_{\mathcal{A}}$ has cardinality at most $\mathfrak{c}$ for every maximal almost disjoint family $\mathcal{A} \subseteq[\mathbb{N}]^{\omega}$. In particular it is undecidable whether $\beta \mathbb{N}$ is homeomorphic to the Cech-Stone remainder of $K_{\mathcal{A}}$ for any maximal almost disjoint family $\mathcal{A} \subseteq[\mathbb{N}]^{\omega}$.

These results suggest that the class of Banach algebras of the form

$$
\mathscr{B}\left(C_{0}\left(K_{\mathcal{A}}\right)\right) / \mathscr{X}\left(C_{0}\left(K_{\mathcal{A}}\right)\right)
$$

may be sensitive to additional set-theoretic assumptions. To establish that this is indeed the case would require noncommutative counterparts of the above consistency results. The arguments would certainly need to involve the structure of continuous self-maps of $K_{\mathcal{A}}$ and its compactifications as in Proposition 36, not only scalar-valued functions on these spaces. This represents a considerable challenge. However, the ideas of [34] to obtain a one-dimensional corona algebra were successfully adapted to work within ZFC in a stronger, noncommutative $C^{*}$-algebraic context in [21].

The following question arises naturally from the above body of results, including Lemma 48:

Question 50. Is there within ZFC a maximal almost disjoint family $\mathcal{A} \subseteq[\mathbb{N}]^{\omega}$ such that $\mathscr{B}\left(C_{0}\left(K_{\mathcal{A}}\right)\right) / \mathscr{X}\left(C_{0}\left(K_{\mathcal{A}}\right)\right)$ is one-dimensional?

Mrówka's original families of [45] are maximal, and the consistently existing families of [34] can also be constructed maximal. By contrast, our construction 
does not give maximality. Indeed, if $\mathcal{A} \subseteq\left[\mathbb{N}^{\omega}\right.$ is the family of [45], the CechStone remainder of $K_{\mathcal{A}}$ is a singleton, so the corona algebra of $C_{0}\left(K_{\mathcal{A}}\right)$ is onedimensional, whereas the family $\mathcal{A}$ from Theorem 2 fails this property because, by the nonmaximality of $\mathcal{A}$, some nonzero elements of the corona algebra correspond to operators with separable range, as explained in Remark 49.

A set $B \in[\mathbb{N}]^{\omega}$ is called a partitioner of an almost disjoint family $\mathcal{A} \subseteq[\mathbb{N}]^{\omega}$ if, for every $A \in \mathcal{A}$, either $A \backslash B$ or $A \cap B$ is finite. A partitioner $B$ partitions the family $\mathcal{A}$ into two parts, namely $\mathcal{A}_{B}=\left\{A \in \mathcal{A}: A \backslash B \in[\mathbb{N}]^{<\omega}\right\}$ and its complement $\left\{A \in \mathcal{A}: A \cap B \in[\mathbb{N}]^{<\omega}\right\}$. This in turn partitions the space $K_{\mathcal{A}}$ into two parts:

$F=\left\{x_{n}: n \in B\right\} \cup\left\{y_{A}: A \in \mathcal{A}_{B}\right\} \quad$ and $\quad\left\{x_{n}: n \in \mathbb{N} \backslash B\right\} \cup\left\{y_{A}: A \in \mathcal{A} \backslash \mathcal{A}_{B}\right\}$,

both of which are closed. Hence the indicator function $1_{F}$ is continuous, so it induces a multiplication operator $M_{1_{F}} \in \mathscr{B}\left(C_{0}\left(K_{\mathcal{A}}\right)\right.$ ), which is clearly a projection. Therefore it decomposes $C_{0}\left(K_{\mathcal{A}}\right)$ into the direct sum of two closed subspaces. Lemma 21 shows that these two subspaces are nonseparable if and only if both $\mathcal{A}_{B}$ and its complement are uncountable.

Partitioners are well studied in the literture (see, e.g., [6]), and almost disjoint families with "few partitioners" were already constructed in the 1940s by Luzin [41], who found an almost disjoint family $\mathcal{A} \subseteq[\mathbb{N}]^{\omega}$ which does not admit any partitioner $B$ such that both $\mathcal{A}_{B}$ and its complement are uncountable. Note that our almost disjoint family $\mathcal{A}$ from Theorem 2 shares this property because $C_{0}\left(K_{\mathcal{A}}\right)$ cannot be decomposed into the direct sum of two nonseparable, closed subspaces. A well-known property of Mrówka's family $\mathcal{A}$ is that it does not admit any partitioner $B$ such that both $\mathcal{A}_{B}$ and its complement are infinite. In fact, Lemma 48 implies that no maximal almost disjoint family $\mathcal{A} \subseteq[\mathbb{N}]^{\omega}$ admits a partitioner $B$ such that either $\mathcal{A}_{B}$ or its complement is countably infinite.

Other results which may help answer Question 46 address the isomorphic classification and the structure of complemented subspaces of $C_{0}\left(K_{\mathcal{A}}\right)$ for an uncountable, almost disjoint family $\mathcal{A} \subseteq[\mathbb{N}]^{\omega}$. Marciszewski and Pol [42] have shown that there are $2^{\mathfrak{c}}$ nonisomorphic Banach spaces of this form. Further, assuming Martin's axiom and the negation of $\mathrm{CH}$, we have the following two results concerning an almost disjoint family $\mathcal{A} \subseteq[\mathbb{N}]^{\omega}$ of uncountable cardinality strictly smaller than $\mathfrak{c}$ :

- The Banach space $C_{0}\left(K_{\mathcal{A}}\right)$ has "many" decompositions as a direct sum of two nonseparable, closed subspaces (see [34]).

- The Banach spaces $C_{0}\left(K_{\mathcal{A}}\right)$ and $C_{0}\left(K_{\mathcal{B}}\right)$ are isomorphic whenever $\mathcal{B} \subseteq[\mathbb{N}]^{\omega}$ is an almost disjoint family of the same cardinality as $\mathcal{A}$. In particular, the Banach space $C_{0}\left(K_{\mathcal{A}}\right)$ is isomorphic to its square (see the recent paper [9] of Cabello Sánchez, Castillo, Marciszewski, Plebanek and Salguero-Alarcón).

\section{REFERENCES}

1. F. Albiac, N. Kalton, Topics in Banach space theory. Second edition. With a foreword by Gilles Godefroy. Graduate Texts in Mathematics, 233. Springer, 2016.

2. D. Alspach, Y. Benyamini, Primariness of spaces of continuous functions on ordinals. Israel J. Math. 27 (1977), 64-92.

3. P. Alexandroff, P. Urysohn, Memoire sur les espaces topologiques compacts dedie a Monsieur D. Egoroff, 1929.

4. S. Argyros, R. Haydon, A hereditarily indecomposable $\mathscr{L}_{\infty}$-space that solves the scalar-pluscompact problem. Acta Math. 206 (2011), no. 1, 1-54.

5. S. Argyros, T. Raikoftsalis, Banach spaces with a unique nontrivial decomposition. Proc. Amer. Math. Soc. 136 (2008), no. 10, 3611-3620. 
6. J. Baumgartner, M. Weese, Partition algebras for almost-disjoint families. Trans. Amer. Math. Soc. 274 (1982), no. 2, 619-630.

7. E. Berkson, H. Porta, Representations of $\mathfrak{B}(X)$. J. Funct. Anal. 3 (1969), 1-34.

8. C. Bessaga, A. Pełczyński, Banach spaces non-isomorphic to their Cartesian squares. I. Bull. Acad. Polon. Sci. Ser. Sci. Math. Astr. Phys. 8 (1960), 77-80.

9. F. Cabello Sánchez, J. Castillo, W. Marciszewski, G. Plebanek, A. Salguero-Alarcón, Sailing over three problems of Koszmider, arXiv:1910.07273.

10. H. G. Dales, A discontinuous homomorphism from $C(X)$. Amer. J. Math. 101 (1979), no. 3, 647-734.

11. H. G. Dales, Banach algebras and automatic continuity. London Math. Soc. Monogr. Ser. 24, Clarendon Press, Oxford, 2000.

12. H. G. Dales, T. Kania, T. Kochanek, P. Koszmider, N. J. Laustsen, Maximal left ideals of the Banach algebra of bounded operators on a Banach space. Studia Math. 218 (2013), 245-286.

13. H. G. Dales, R. J. Loy, G. A. Willis, Homomorphisms and derivations from $\mathscr{B}(E)$. J. Funct. Anal. 120 (1994), 201-219.

14. J. Diestel, Grothendieck spaces and vector measures. Vector and operator valued measures and applications (Proc. Sympos., Alta, Utah, 1972), 97-108. Academic Press, New York, 1973.

15. J. Diestel, J. Uhl, Vector measures. With a foreword by B. J. Pettis. Mathematical Surveys, No. 15. American Mathematical Society, Providence, R.I., 1977.

16. A. Dow, Large compact separable spaces may all contain $\beta \mathbb{N}$. Proc. Amer. Math. Soc. 109 (1990), 275-279.

17. I. S. Edelstein, B. S. Mityagin, Homotopy type of linear groups of two classes of Banach spaces. Funct. Anal. Appl. 4 (1970), 221-231.

18. F. van Engelen, K. Kunen, A. Miller, Two remarks about analytic sets. Set theory and its applications (Toronto, ON, 1987), 68-72, Lecture Notes in Math., 1401. Springer, Berlin, 1989.

19. R. Engelking, General topology. Translated from the Polish original by the author. Second edition. Sigma Series in Pure Mathematics, 6. Heldermann Verlag, Berlin, 1989.

20. J. Esterle, Injection de semi-groupes divisibles dans des algèbres de convolution et construction d'homomorphismes discontinus de C $(K)$. Proc. London Math. Soc. (3) 36 (1978), no. 1, 59-85.

21. S. Ghasemi, P. Koszmider, An extension of compact operators by compact operators with no nontrivial multipliers. J. Noncommut. Geom. 12 (2018), no. 4, 1503-1529.

22. T. Gowers, B. Maurey, The unconditional basic sequence problem. J. Amer. Math. Soc. 6 (1993), 851-874.

23. F. Hernández-Hernández, M. Hrušák, Topology of Mrówka-Isbell spaces. Pseudocompact topological spaces, 253-289, Dev. Math., 55. Springer, Cham, 2018.

24. M. Hrušák, Almost disjoint families and topology. Recent progress in general topology. III, 601-638. Atlantis Press, Paris, 2014.

25. R. James, A non-reflexive Banach space isometric with its second conjugate space. Proc. Nat. Acad. Sci. U.S.A. 37 (1951), 174-177.

26. B. E. Johnson, Continuity of homomorphisms of algebras of operators. J. London Math. Soc. 42 (1967), 537-541.

27. W. Johnson, J. Lindenstrauss, Some remarks on weakly compactly generated Banach spaces. Israel J. Math. 17 (1974), 219-230.

28. T. Kania, T. Kochanek, The ideal of weakly compactly generated operators acting on a Banach space. J. Op. Th. 71 (2014), no. 2, 455-477.

29. T. Kania and N. J. Laustsen, Uniqueness of the maximal ideal of the Banach algebra of bounded operators on $C\left(\left[0, \omega_{1}\right]\right)$. J. Funct. Anal. 262 (2012), 4831-4850.

30. T. Kania, N. J. Laustsen, Ideal structure of the algebra of bounded operators acting on a Banach space. Indiana Univ. Math. J. 66 (2017), no. 3, 1019-1043.

31. T. Kania, P. Koszmider, N. J. Laustsen, A weak*-topological dichotomy with applications in operator theory. Trans. London Math. Soc. 1 (2014), no. 1, 1-28.

32. A. Kechris, Classical descriptive set theory. Graduate Texts in Mathematics, 156. SpringerVerlag, New York, 1995.

33. P. Koszmider, Banach spaces of continuous functions with few operators. Math. Ann. 330 (2004), no. 1, 151-183.

34. P. Koszmider, On decompositions of Banach spaces of continuous functions on Mrówka's spaces. Proc. Amer. Math. Soc. 133 (2005), no. 7, 2137-2146. 
35. P. Koszmider, A survey on Banach spaces $C(K)$ with few operators. Rev. R. Acad. Cienc. Exactas Fís. Nat. Ser. A Mat. RACSAM 104 (2010), no. 2, 309-326.

36. P. Koszmider, P. Zieliński, Complementation and decompositions in some weakly Lindelöf Banach spaces. J. Math. Anal. Appl. 376 (2011), 329-341.

37. J. Kulesza, R. Levy, Separation in $\Psi$-spaces. Topology Appl. 42 (1991), 101-107.

38. N. J. Laustsen, Maximal ideals in the algebra of operators on certain Banach spaces. Proc. Edinburgh Math. Soc. 45 (2002), 523-546.

39. J. Lindenstrauss, L. Tzafriri, Classical Banach Spaces I: Sequence Spaces. Springer Verlag, 1977.

40. R. J. Loy, G. A. Willis, Continuity of derivations on $\mathscr{B}(E)$ for certain Banach spaces E. J. London Math. Soc. 40 (1989), 327-346.

41. N. N. Luzin, On subsets of the series of natural numbers. Izvestiya Akad. Nauk SSSR. Ser. Mat. 11 (1947), 403-410.

42. W. Marciszewski, R. Pol, On Banach spaces whose norm-open sets are $F_{\sigma}$-sets in the weak topology. J. Math. Anal. Appl. 350 (2009), no. 2, 708-722.

43. B. Maurey, Banach spaces with few operators. In Handbook of the Geometry of Banach Spaces. Edited by W. B. Johnson and J. Lindenstrauss. Vol. 2 (2003), 1247-1297.

44. P. Motakis, D. Puglisi, D. Zisimopoulou, A hierarchy of separable commutative Calkin algebras. Indiana Univ. Math. J. 65 (2016), no. 1, 39-67.

45. S. Mrówka, Some set-theoretic constructions in topology. Fund. Math. 94 (1977), no. 2, 83-92.

46. C. Ogden, Homomorphisms from B $\left(C\left(\omega_{\eta}\right)\right)$. J. London Math. Soc. 54 (1996), 346-358.

47. A. Pełczyński, On strictly singular and strictly cosingular operators. I. Strictly singular and strictly cosingular operators in $C(S)$-spaces. Bull. Acad. Pol. Sci. 13 (1965), 31-37.

48. A. Pełczyński, Z. Semadeni, Spaces of continuous functions. III. Spaces $C(\Omega)$ for $\Omega$ without perfect subsets. Studia Math. 18 (1959), 211-222.

49. G. Plebanek, A construction of a Banach space $C(K)$ with few operators, Topology Appl. 143 (2004), 217-239.

50. C. J. Read, Discontinuous derivations on the algebra of bounded operators on a Banach space. J. London Math. Soc. 40 (1989), 305-326.

51. W. Rudin, Continuous functions on compact spaces without perfect subsets. Proc. Amer. Math. Soc. 8 (1957), 39-42.

52. W. Schachermayer, On some classical measure-theoretic theorems for non-sigma-complete Boolean algebras. Diss. Math. (Rozprawy Mat.) 214, 1982.

53. Z. Semadeni, Banach spaces non-isomorphic to their Cartesian squares. II. Bull. Acad. Polon. Sci. Ser. Sci. Math. Astr. Phys. 8 (1960), 81-84.

54. Z. Semadeni, Banach spaces of continuous functions. Vol. I. Monografie Matematyczne, Tom 55. PWN-Polish Scientific Publishers, Warsaw, 1971.

55. S. Shelah, A Banach space with few operators. Israel J. Math. 30 (1978), 181-191.

56. S. Shelah, J. Steprāns, A Banach space on which there are few operators. Proc. Amer. Math. Soc. 104 (1988), 101-105.

57. M. Tarbard, Hereditarily indecomposable, separable $\mathscr{L}_{\infty}$ Banach spaces with $\ell_{1}$ dual having few but not very few operators. J. London Math. Soc. (2) 85 (2012), no. 3, 737-764.

58. H. M. Wark, A non-separable reflexive Banach space on which there are few operators. J. London Math. Soc. 64 (2001), 675-689.

59. H. M. Wark, A non-separable uniformly convex Banach space on which there are few operators. Studia Math. 241 (2018), 241-256.

60. A. Wilansky, Summability through functional analysis. Mathematics Studies 85, NorthHolland, 1984.

61. G. Willis, Compressible operators and the continuity of homomorphisms from algebras of operators. Studia Math. 115 (1995), 251-259.

(P. Koszmider) Institute of Mathematics, Polish Academy of Sciences, ul. ŚniaDECKICH 8, 00-656 WARsZaWA, POland

Email address: piotr.koszmider@impan.pl

(N. J. Laustsen) Department of Mathematics and Statistics, Fylde College, Lancaster University, Lancaster, LA1 4YF, United Kingdom

Email address: n.laustsen@lancaster.ac.uk 